\title{
Non-Intrusive In-Situ Requirements Monitoring of Embedded System
}

\author{
MINJUN SEO and ROMAN LYSECKY, University of Arizona
}

\begin{abstract}
Accounting for all operating conditions of a system at the design stage is typically infeasible for complex systems. Monitoring and verifying system requirements at runtime enable a system to continuously and introspectively ensure the system is operating correctly in the presence of dynamic execution scenarios. In this article, we present a requirements-driven methodology enabling efficient runtime monitoring of embedded systems. The proposed approach extracts a runtime monitoring graph from system requirements specified using UML sequence diagrams. Non-intrusive, on-chip hardware dynamically monitors the system execution, verifies the execution adheres to the requirements model, and in the event of a failure provides detailed information that can be analyzed to determine the root cause. Using case studies of an autonomous vehicle and pacemaker prototypes, we analyze the relationship between event coverage, detection rate, and hardware requirements
\end{abstract}

\section{CCS Concepts: • Computer systems organization $\rightarrow$ Embedded systems;}

Additional Key Words and Phrases: Runtime requirements monitoring, embedded systems, non-intrusive system monitoring

\section{ACM Reference format:}

Minjun Seo and Roman Lysecky. 2018. Non-Intrusive In-Situ Requirements Monitoring of Embedded System. ACM Trans. Des. Autom. Electron. Syst. 23, 5, Article 58 (August 2018), 27 pages.

https://doi.org/10.1145/3206213

\section{INTRODUCTION}

Defining and verifying system requirements are critical challenges in embedded systems. Verification methods include both test-based and formal verification methods (Whalen et al. 2007) and commonly account for as much as $75 \%$ of the total system design effort (Hailpern and Santhanam 2002). Even with this considerable effort at design-time, runtime verification remains essential for many embedded systems. This is due to both the system complexity and the highly dynamic nature of applications and the environments in which they operate. Exhaustively testing for all possible data inputs, application configurations, and environmental conditions is at best infeasible or cost prohibitive and at worst impossible. Notable examples in which design-time verification was insufficient to ensure system correctness at runtime include the Patriot Missile that failed to intercept the intended target due to an arithmetic error (Skeel 1992), the Mars Pathfinder that malfunctioned due to a scheduling problem (Jones 1997), the Therac-25 in which previously existing code was poorly reused (Leveson and Turner 1993), and the ADIRU component used in the Boeing 777 and

This research was partially supported by the National Science Foundation under Grant CNS-1563652.

Authors' address: M. Seo and R. Lysecky, Department of Electrical and Computer Engineering, University of Arizona, 1230 E. Speedway Blvd., Tucson, AZ 85721; emails: mjseo@email.arizona.edu, rlysecky@ece.arizona.edu.

Permission to make digital or hard copies of all or part of this work for personal or classroom use is granted without fee provided that copies are not made or distributed for profit or commercial advantage and that copies bear this notice and the full citation on the first page. Copyrights for components of this work owned by others than ACM must be honored. Abstracting with credit is permitted. To copy otherwise, or republish, to post on servers or to redistribute to lists, requires prior specific permission and/or a fee. Request permissions from Permissions@acm.org.

(C) 2018 ACM 1084-4309/2018/08-ART58 \$15.00

https://doi.org/10.1145/3206213 
Airbus A330 that incorrectly assumed only a single sensor could fail, resulting in an invalid sensor reading being returned as valid (Bonakdarpour et al. 2011; Macaulay 1992).

Embedded systems present unique challenges in monitoring correctness. The intricate integration of hardware and software means runtime monitoring methods must consider both hardware and software aspects (Delgado et al. 2004), which increases the monitoring complexity. This also prevents the use of traditional methods to observe, monitor, or control the state of these components at runtime. As such, ensuring systems operate correctly in situ is a significant challenge. Thus, there is a need for methods that continually verify the correctness of a system's execution at runtime within the deployed system, especially for safety-critical and life-critical systems, such as autonomous vehicles and medical devices.

In-situ runtime monitoring and verification of embedded systems can enable the system to introspectively ensure the system is operating correctly in the presence of dynamic environment, rapidly detect failures or deviations in the expected system execution, and provide detailed execution traces to find the root cause of those unexpected behaviors. Importantly, runtime verification can ensure embedded systems adhere to system requirements in the presence of unknown environmental, physical, and operating conditions. However, due to strict resource constraints, runtime monitoring should be non-intrusive, as intrusive monitoring methods would perturb a system's behavior and result in negative side effects. For example, timing changes from an intrusive monitoring system may change the system execution such that synchronization and scheduling issues are introduced, which in the worst case can lead to system failure.

In this article, we present a requirements-driven methodology enabling non-intrusive in-situ requirements monitoring (NIRM) of embedded systems using non-intrusive hardware. The NIRM approach extracts and synthesizes a runtime monitoring graph from system requirements specified using UML sequence diagrams. UML sequence diagram has been widely used in software development and can be used to model both synchronous and asynchronous operation as well as interactions between software and hardware components. UML sequence diagrams are leveraged to extract systems requirements without requiring distinct and separate requirements models. We present a formal Runtime Monitoring Graph (RMG) model that compactly represents system requirements extracted from the sequence diagrams and enables efficient runtime verification using non-intrusive hardware. We present the design of non-intrusive, on-chip hardware that monitors the system execution at runtime to ensure the system execution matches the requirements model. Critically, the on-chip hardware component incurs zero performance overhead and requires no internal processor modifications. While the presented NIRM hardware could be used to monitor and verify the execution of hardware components, in this article, we focus only on monitoring and verifying software execution. Supporting evaluation metrics are proposed to enable optimizing requirements coverage subject to hardware constraints.

\section{RELATED WORK}

\subsection{Software-Based Runtime Monitoring and Verification Methods}

Software-based runtime monitoring and verification execute additional software to observe system events and ensure the system execution adheres to the requirements model or ensures system properties hold true. However, the instrumentation and analysis of software-based approaches incur significant runtime overheads.

DTrace (Beauchamp and Weston 2008) is a dynamic tracing tool that can be utilized to trace various system behaviors, e.g., stack overflow, heap overflow, and system call execution without help of hardware, but it carries significant runtime overheads that can negatively impact the system execution. 
Periodic sampling can reduce the overhead of software-based requirements verification methods. Bonakdarpour et al. (2011) demonstrated that optimizing the sampling frequency of events can reduce the runtime overhead while ensuring sufficient requirements coverage. Although periodic sampling reduces the overhead, these approaches are still intrusive and may change the execution behavior, making verification of hard real-time constraints difficult.

Time-triggered runtime verification (TTRV) is an approach that seeks to minimize the software overhead of runtime verification for multicore systems (Navabpour et al. 2015). During the design process, TTRV seeks to find an optimal mapping of software components to processors cores and an optimal configuration of monitoring frequency to minimize overhead of the monitoring and verification software. While TTRV reduces the overhead by up to $34.1 \%$, compared to event-triggered monitoring, the runtime monitoring and verification software still incurs a $7 \%$ performance overhead and a $51 \%$ memory overhead.

EgMon reduces the software overhead for runtime verification by focusing on monitoring messages transmitted between components, specifically monitoring messages broadcast over a CAN bus and verifying requirements defined using bounded metric temporal logic (Kane et al. 2015). EgMon uses a separate device on the CAN bus through USB connection, which reduces the performance overhead for the system but does not eliminate it, due to the added delay of the EgMon device.

Chai et al. presented a runtime verification system that utilizes live sequence charts (LSCs) (Chai and Schlingloff 2014). LSCs are similar to UML sequence diagrams and enable support modeling multiple system behaviors, conditional execution sequences, and activation timing (Brill et al. 2004). The approach presented by Chai et al. concatenates several LSCs to define the possible states of the system that can be examined, and then transforms the concatenated LSC into linear temporal logic (LTL). Although LSC can be transformed to LTL, the number of events in the resulting LTL can be exponential. Also, LTL model checking provides powerful verification method, it would not be the best method to implement monitoring using hardware.

Copilot is a compiler-assisted approach that automatically instruments a software binary with custom verification code compiled from a requirements specification language (Pike et al. 2011). Copilot generates verification code, for which the timing can be statically analyzed. While this approach reduces the effort required to verify hard real-time constraints for the system, any changes in requirements mandate re-verifying that the hard real-time constraints are met.

Although these methods try to minimize or control the overhead, software-based approaches still incur significant runtime overheads that can negatively impact the system execution and present challenges for design-time verification.

\subsection{Hardware-Based Runtime Monitoring and Verification Methods}

Alternatively, hardware-based runtime monitoring can be utilized to dynamically verify that the system implementation adheres to the required system properties. However, most runtime monitoring methods are not specifically targeted at verifying system requirements at runtime but are more geared toward testing and debugging.

JTAG scan chains (Stollon 2011) are a standard method utilized for hardware debugging that allows all registers within a design to be monitored at runtime. However, access to those registers incurs significant overhead, as the system must be entirely halted to access the scan chain.

Trace-based methods for monitoring system events (e.g., CoreSight (Mijat 2010), ChipScope (X. V. Tool 2006), SignalTap (A. V. Tool 2006), and real-time non-intrusive (RTNI) (Fryer 2005)) allow designers to specify a set of signals that can be traced at runtime without affecting the system performance. The RTNI approach utilizes a trace-based approach for tracing specific signals within a microprocessor focused on test, debug, and validation of real-time systems (Fryer 2005). However, 
those trace methods typically require external hardware or dedicated processors to access the traced signals and only allow a small subset of signals to be traced.

Watchdog timers are useful and are a widely used method to recover from system failure (Murphy and Barr 2001). During the correct execution, the system will periodically reset the watchdog timer. In the event that the watchdog timer is not reset at the defined rate, the watchdog assumes the system execution is faulty and takes corrective action, e.g., performs a system reset. While multiple watchdog timers can be utilized, they provide a method for verifying that certain operations are executed at the defined rate but cannot verify complex system requirements.

MAMon (El Shobaki 2002) uses a hardware method and performs monitoring on specific RTOS kernels. The approach sends system level information such as task switches, system calls, and interrupts to a separate host via a parallel port. It does not support detailed software monitoring.

$\mathrm{P} 2 \mathrm{~V}$ is a hardware-based verification method that extends the memory access stage of a MIPS processor to implement a monitor unit (MU) (Lu 2007). The P2V approach synthesizes requirements specified using a subset of property specification language (PSL) to a custom dedicated hardware component integrated with the memory access stage. This approach enables non-intrusive verification but is limited to verifying memory accesses and does not support software updates.

Hard (Zhou et al. 2007) provides a mechanism that can detect thread race by hardware implementation. This can be useful to detect software bugs especially related to thread, which is usually a challenge for programmers. Hard modifies the existing microprocessor to add Bloom filter and area for vector register and also causes performance overhead from $0.1 \%$ to $2.5 \%$ due to increased communication.

AVIO-H (Lu et al. 2007) identifies software bugs caused by atomicity violations. AccessInterleaving (AI) Invariance is a key factor of causing the bugs. AVIO-H extracts AI invariance by training sets and can detect atomicity violations. This approach requires not only additional steps for offline tests and training but also efforts to choose high-quality training data.

DAProf (Nair et al. 2011) utilized hardware to non-intrusively profile the execution of software loops by directly interfacing hardware to the processor using the available trace ports. Our NIRV hardware utilizes a similar method to monitor the system without perturbing the system execution.

Reinbacher et al. automatically synthesize requirements specified in past-time Metric Temporal Logic (ptMTL) to hardware that verifies those requirements over fixed time intervals (Reinbacher et al. 2012). This approach observes communication-based events by monitors either the system bus, network-on-chip, or physical IO, which presents challenges in the types of events that can be monitored and the system-level design process.

Backasch et al. utilize a hidden in-circuit emulator (hidICE) to non-intrusively detect events within the main system and report those events to an external emulator using a dedicated port (Backasch et al. 2013). The runtime verification is then performed using the external emulator, which ensures the main system's execution is unaffected. While this approach is non-intrusive, the external emulator requires significant area and energy overheads. Additionally, the number of events that can be monitored is limited by the bandwidth of the communication port.

Hofmann et al. presented a hybrid monitoring technique that combines software instrumentation to detect events or specific conditions with hardware that monitors those events (Hofmann et al. 1994). Such hybrid approaches provide flexibility such that the instrumentation for detecting events can be easily changed while reducing the runtime overhead of monitoring by offloading the processing of events to hardware. Alternatively, NUVA uses hardware-based instrumentation enabled by custom instructions to detect events and dedicated hardware that verify system requirements specified using parametric FSMs (Nassar et al. 2015). While the use of custom instructions reduces the performance overhead compared to software-based instrumentation, a performance overhead of approximately $5 \%$ is still incurred. 


\subsection{Requirements Models for Runtime Verification}

Numerous models exist for specifying requirements for runtime monitoring and verification. Formal models such as timed automata and linear temporal logic use a mathematical framework to completely define requirements for the entire system. Linear temporal logic (LTL) defines system states and temporal properties of the systems states (Pnueli 1977), which can be verified at runtime to ensure those properties remain true throughout a system's execution. Real-time extensions on LTL such as metric interval temporal logic (MITL) have been proposed to specify real-time requirements supporting a concept of specific time interval for LTL.

The Timed Automata (TA) (Alur 1999) model is another well-known formal modeling approach for specifying how a system operates and modeling the time constraints for transition between system states. TA models are typically used by model checking techniques, primarily at the component level, to evaluate properties such as reachability, safety, and liveness property. However, TA models face challenges in modeling the interactions at the system level (e.g., hardware and integration and interactions).

While both LTL and TA (along with many related formal requirements models) can succinctly specify system requirements and can be used at runtime to verify systems correctness, these modeling techniques are not commonly used by software developers. Integrating the use of LTL or TA in standard software development processes would require developers to maintain multiple system models, which is time-consuming and error prone and would require extensive training (Chai and Schlingloff 2014).

UML sequence diagrams (Rumbaugh et al. 2005), which are one of the UML interaction diagrams, are widely used in software development and can be considered as a standard model for software development. Earlier versions of sequence diagrams had limited support to model numerous execution scenarios, which limited its use for runtime verification. However, the current UML standard's sequence diagrams support modeling multiple scenarios using combined fragments and support both synchronous and asynchronous operations. Notably, sequence diagrams are also used to model system level interaction between components, including both software and hardware components.

UML sequence diagrams have been used for verification, including several approaches that convert the UML sequence diagrams to TA models (David 2003; Firley et al. 1999). While converting UML sequence diagrams to TA models have been shown to be feasible, there are several limitations. One approach converts each sequence diagram to separate TA models (David 2003), which requires later combining those models together, leading to the well-known state explosion problem. Another approach supports converting hierarchical UML models to hierarchical timed automata (David 2003) but still requires multiple TA models. Overall, TA models are not ideal for non-intrusive runtime monitoring and verification due to the hardware resources that would be required to support system-level TA models.

\section{NON-INTRUSIVE IN-SITU REQUIREMENT MONITORING METHODOLOGY}

The NIRM methodology for in-situ requirements monitoring consists of (1) a design-time methodology for system requirements specification and analysis and (2) in-situ, non-intrusive hardware for monitoring and verifying requirements at runtime within the deployed system.

Figure 1(a) presents the NIRM methodology consisting of (1) initial software development and UML sequence diagrams, (2) automated synthesis of requirements from the UML sequence diagrams, (3) runtime monitoring graph (RMG) generation, (4) RMG analysis and optimization, (5) RMG configuration generation and binary integration, and (6) in-situ, non-intrusive monitoring of system execution. 


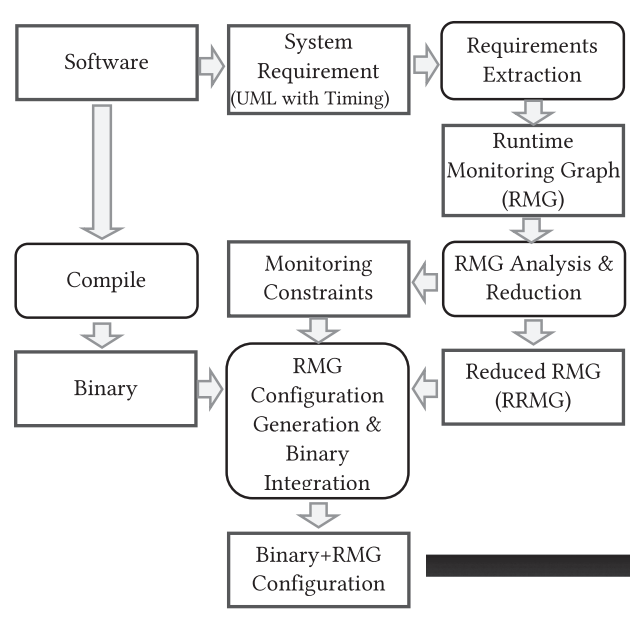

(a)

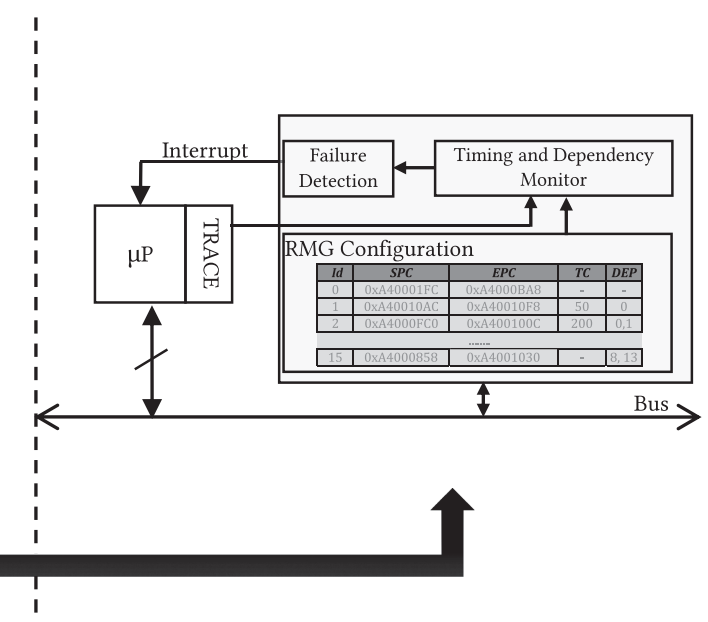

(b)

Fig. 1. Overview of (a) design methodology for in-situ monitoring and (b) integration of non-intrusive runtime monitoring hardware with processor.

\section{REQUIREMENT SPECIFICATION AND EXTRACTION FROM UML SEQUENCE DIAGRAM}

Sequence diagrams capture the execution flow of programs for individual tasks, communication between tasks, and interfaces with hardware components for different use case scenarios. These sequence diagrams are a standard approach used within the design and analysis of software. Realtime UML (Bruce 1999) and the UML profile for Modeling and Analysis of Real-Time and Embedded systems (MARTE) (Gérard and Selic 2008) provide extensions to UML sequence diagrams specifying real-time scheduling constraints. However, these UML models primarily focus on facilitating schedulability analysis and performance analysis but not in-situ requirements monitoring.

To support the specification of timing requirements for complex system operations, the proposed approach utilizes extensions to real-time UML sequence diagrams that incorporate timing annotations to specifying timing constraints for individual operations and sequence of operations.

Figure 2(a) presents an example UML sequence diagram with timing annotations for an image capture and storage interaction within a digital camera subsystem. The system consists of four objects, namely the Camera, ImageProcessor, Button, and ENV objects, in which each object represents a separate application task. The sequence diagram specifies the order of interactions between those components, the names of message that are passed between components, and the timing requirements. When the button is pressed, the buttonPressed() message will be passed to the ENV object, which will in turn send a doTakePicture() message to the Camera object. Note that the ENV object represents the system environment, including the operating system, interrupt service routines, and so on (Douglass 2014). The Camera object will interface with the camera sensor to capture an image storing that image within a hardware buffer. The ImageProcessor object is an application task that executes after the image capture has completed to read the image from the hardware buffer, process the image, and, finally, send a imageUpdate() message to the ENV object, in which the final image is passed as a parameter of that message.

Timing requirements for scheduling tasks and specific operations are specified using UML's annotations, such that existing tools for creating sequence diagrams can be directly utilized. Scheduling constraints for the Camera and ImageProcessor object define when the tasks should be 

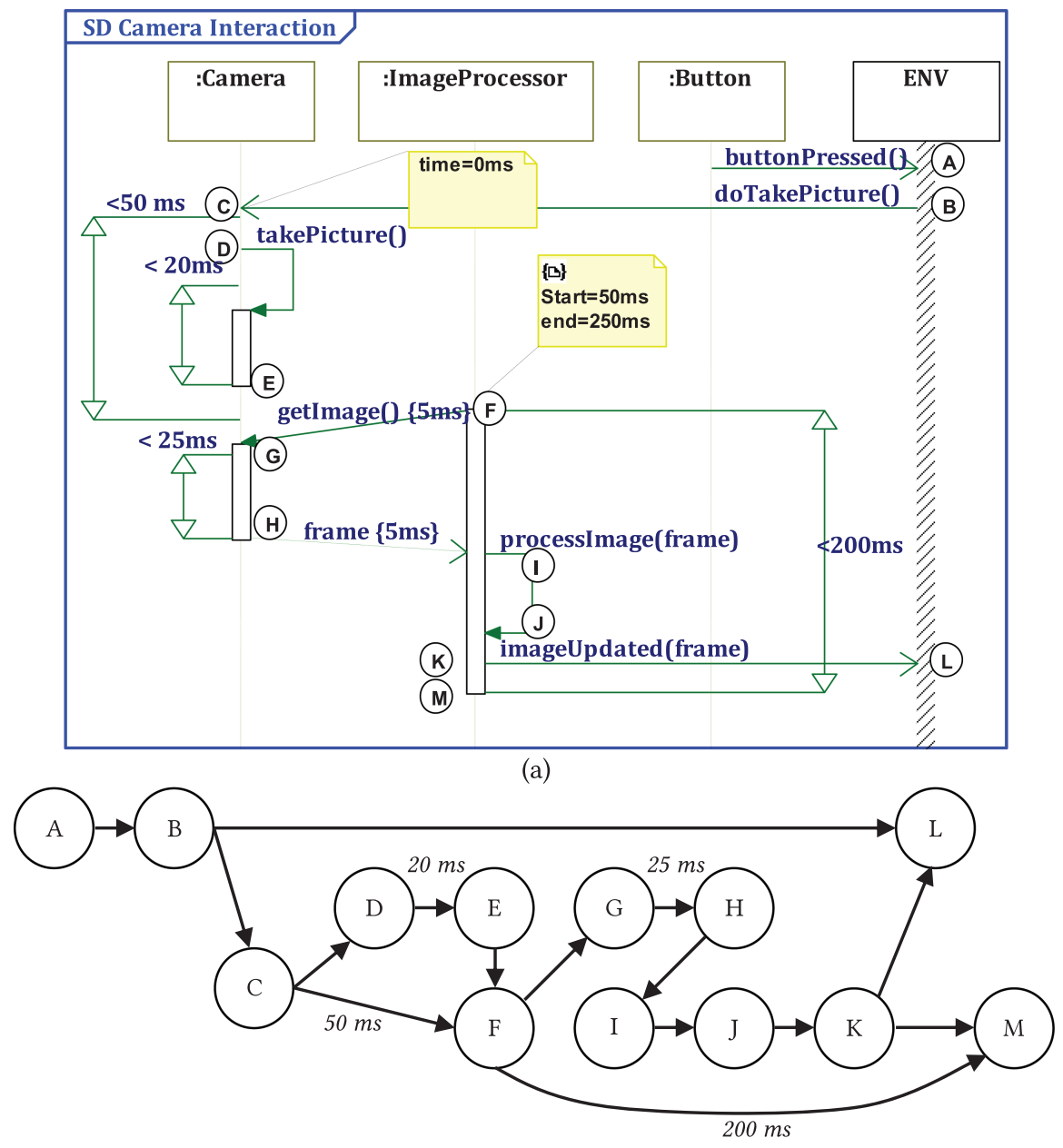

(b)

Fig. 2. (a) Overview of runtime verification graph extraction showing (a) definition of verification events superimposed on the UML sequence diagram and (b) resulting runtime verification graph.

scheduled for execution once the doTakePicture message is sent, where the Camera object should be immediately scheduled for execution and the ImageProcessor object should be scheduled for execution 50ms later. Additionally, the ImageProcessor is defined with an execution deadline constraint of $250 \mathrm{~ms}$. Timing requirements are also defined for individual operations within the sequence diagram. For example, the total execution time for transferring the image data from the Camera to the ImageProcessor should be less than $25 \mathrm{~ms}$, shown in the sequence diagram with the timing annotation between the getImage() message sent to the Camera object and the frame sent back to the ImageProcessor.

\section{RUNTIME MONITORING GRAPH (RMG)}

We define a runtime monitoring graph, extracted from the sequence diagrams, that captures all requirements that can be monitored. The RMG defines the set of events that must be observed 
at runtime, the correct execution order for those events, and timing constraints between events, where applicable. All messages and causality of messages (i.e., sender and receiver) within the sequence diagrams are considered system events. Figure 2(a) illustrates the extraction of events within the UML sequence diagram for the sensor data acquisition of the digital camera subsystem, which represents an initial 1:1 mapping between a single sequence diagram and RMG. The RMG enables the extraction and integration of events and timing information from multiple sequence diagrams into a single model, which can leverage hierarchy to efficiently represent system requirements, although the automated identification of hierarchy is left as future work.

Definition 1. An RMG is a tuple $G=V_{G}, E_{G}$, where

- $\mathrm{V}_{\mathrm{G}}$ is a finite set of vertices, where each vertex represents a system event.

- $\mathrm{E}_{\mathrm{G}}$ is a set edges representing dependencies between vertices. Each edge $E=\mathrm{V}_{\mathrm{G}} \rightarrow \mathrm{V}_{\mathrm{G}}{ }^{\prime}$ represents a dependency between two events $\mathrm{V}_{\mathrm{G}}$ and $\mathrm{V}_{\mathrm{G}}{ }^{\prime}$. $\mathrm{V}_{\mathrm{G}}$ is the source event and $\mathrm{V}_{\mathrm{G}}{ }^{\prime}$ is the sink event.

- $\mathrm{V}_{\mathrm{G}} \stackrel{\text { time }}{\rightarrow} \mathrm{V}_{\mathrm{G}}{ }^{\prime}$ specifies an edge with a worst-case execution time (WCET) constraint between two events if a time constraint exists between $\mathrm{V}_{\mathrm{G}}$ and $\mathrm{V}_{\mathrm{G}}{ }^{\prime}$.

Figure 2(b) shows the resulting RMG extracted from the UML sequence diagram in Figure 2(a), highlighting the extracted events, execution order dependencies between those events, and timing requirements for a subset of those events. For example, the edge $G \rightarrow H$ indicates event $G$ should occur before event $H$, and the time constraint $G \stackrel{25 m s}{\rightarrow} H$ indicates event $H$ should occur within $25 \mathrm{~ms}$ after event $G$.

\section{RMG EVALUATION METRICS, ANALYSIS, AND REDUCTION}

\subsection{RMG Evaluation Metrics}

For complex systems, supporting all possible RMG events within the NIRM hardware may be infeasible due to area and energy constraints. Thus, event selection methods will be needed to select those events within the full RMG to satisfy area, energy, and coverage constraints. Given the complete RMG for a system, a reduced RMG (RRMG) is defined as a subset of events within the RMG that may be monitored given a constraint on the number of events supported in hardware.

To evaluate requirements coverage in the RRMG, three evaluation metrics are considered: execution path coverage (EPC), timing path coverage (TPC), and average path coverage (APC). The path coverage metrics integrate the percentage of paths and precision with which paths in the full RMG are covered in the RRMG. A path precision index (PPI) measures how much information is maintained in the RRMG (i.e., how closely a path in the RRMG matches the original path in the RMG). We separately calculate an execution path precision index (EPPI) and a timing path precision index (TPPI).

Execution Path Coverage (EPC). For a path, $P_{i}$, in the RRMG, the EPPI calculates the number of events in the path compared to the number of events in the closest matching path within the original RMG. EPC calculates the average EPPI for all paths within the RRMG,

$$
\begin{gathered}
\operatorname{EPPI}\left(P_{i}\right)=\frac{\text { NumEvents }\left(P_{i}\right)}{\text { NumEvents (NearestPath } \left.\left(R M G, P_{i}\right)\right)} \\
\operatorname{EPC}(R R M G)=\frac{\sum_{P_{n} \in R R M G} \operatorname{EPPI}\left(P_{n}\right)}{\text { NumPaths }(R R M G)} * 100 .
\end{gathered}
$$

NumEvents() is the number of events in a path and NumPaths() is the number of paths in an RMG or RRMG. 
Timed Path Coverage. TPC is the percentage of timing information within the full RMG that is covered within the RRMG. Timing requirements are specified as time constraints on edges between vertices. The TPPI calculates the number of timed events within a path compared to the original path in the RMG. The TPC calculates the average TPPI for all timed paths within the RRMG,

$$
\begin{gathered}
\operatorname{TPPI}\left(P_{i}\right)=\frac{\text { NumTimedEvents }\left(P_{i}\right)}{\text { NumTimedEvents (NearestPath } \left.\left(R M G, P_{i}\right)\right)} \\
\operatorname{TPC}(R R M G)=\frac{\sum_{P_{n} \in R R M G} \operatorname{TPPI}\left(P_{n}\right)}{\text { NumTimedPaths }(R R M G)} * 100 .
\end{gathered}
$$

NumTimedEvents() calculates the number of timed events in a path and NumTimedPaths() calculates the number of timed paths in RMG or RRMG.

Average Path Coverage (APC). As an RMG encapsulates both execution sequence and timing requirements, the average path coverage (APC) is calculated as the average of the EPC and TPC:

$$
A P C(R R M G)=\frac{E P C(R R M G)+T P C(R R M G)}{2} .
$$

\subsection{RMG Reduction}

The NIRM approach employs greedy optimization algorithms that iteratively refine the RMG by removing or adding events with the goal of maximizing the APC. We present two greedy algorithms, namely a greedy iterative algorithm presented in Algorithm 1 and a multi-phase iterative algorithm presented in Algorithm 2. Additionally, we compare these greedy algorithms to a simulated annealing based optimization approach. The following details the three optimization algorithms considered.

Greedy Iterative Algorithm. The greedy iterative algorithm (Algorithm 1) iteratively removes one event until the RRMG fits within the hardware constraint defined as the maximum number of events (MaxEvents) supported by the hardware monitor. At each iteration, the algorithm evaluates all candidate RRMG's ( $R M G C$ 's) that can be created by removing one event. For each of the RMGCs, the APC is evaluated, and the RMGC with the maximum APC is selected. Thus, the algorithm iteratively removes one event at a time, removing the events that result in the smallest requirements coverage loss, measured by the APC. Figure 3 shows two RRMGs for the autonomous vehicle system presented in Section 9. Figure 3(a) is an RRMG using a maximum event constraint of 17 events. In reducing the RMG, the iterative greedy optimization algorithm removes 5 events, 8 sequence dependencies, and no timing constraints, resulting in an APC and detection rate of $91.8 \%$ and $100 \%$, respectively. Figure 3(b) is an RRMG using a maximum event constraint of 13 events. The reduction algorithm removes 9 events, 13 dependencies, and 1 timing constraint, resulting in an APC of $88.9 \%$ but still achieving a $100.00 \%$ detection rate in practice. Note that the reduction algorithm's tendency is to not reduce timing related events to achieve a higher APC.

Multi-phase Iterative Algorithm. The multi-phase iterative algorithm shown in Algorithm 2 first uses the greedy iterative algorithm to determine an initial RRMG that meets the specified maximum number of events followed by a refinement phase. RRMGI is the initial RRMG determined using Algorithm 1. The refinement phase then tries replacing each event in the RRMGI with an unselected event in the full RMG. For each replacing each event in RRMGI, the algorithm tries all possible candidate event (ce) in the RMG, replaces the event with the candidate event yielding the highest APC. If no APC increase is possible, then the event is not replaced.

Simulated Annealing Algorithm. To compare the efficiency and optimality of the greedy iterative and multiphase iterative algorithms, we additionally compare to a simulated annealing based 


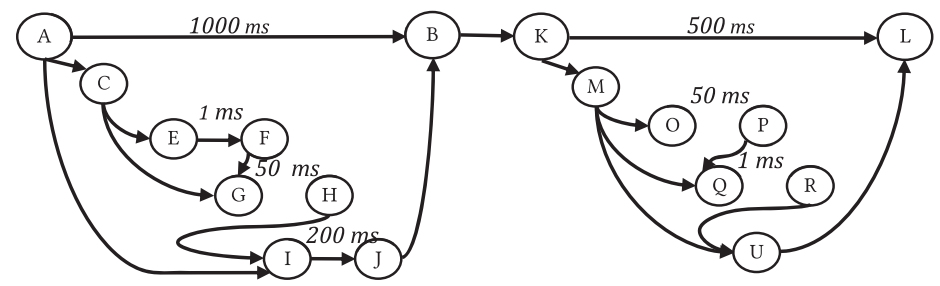

(a)

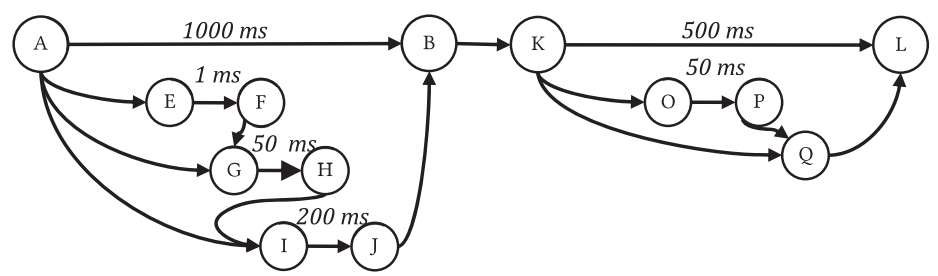

(b)

Fig. 3. Optimized RRMGs using maximum event constraints of (a) 17 events and (b) 13 events using the iterative greedy algorithm.

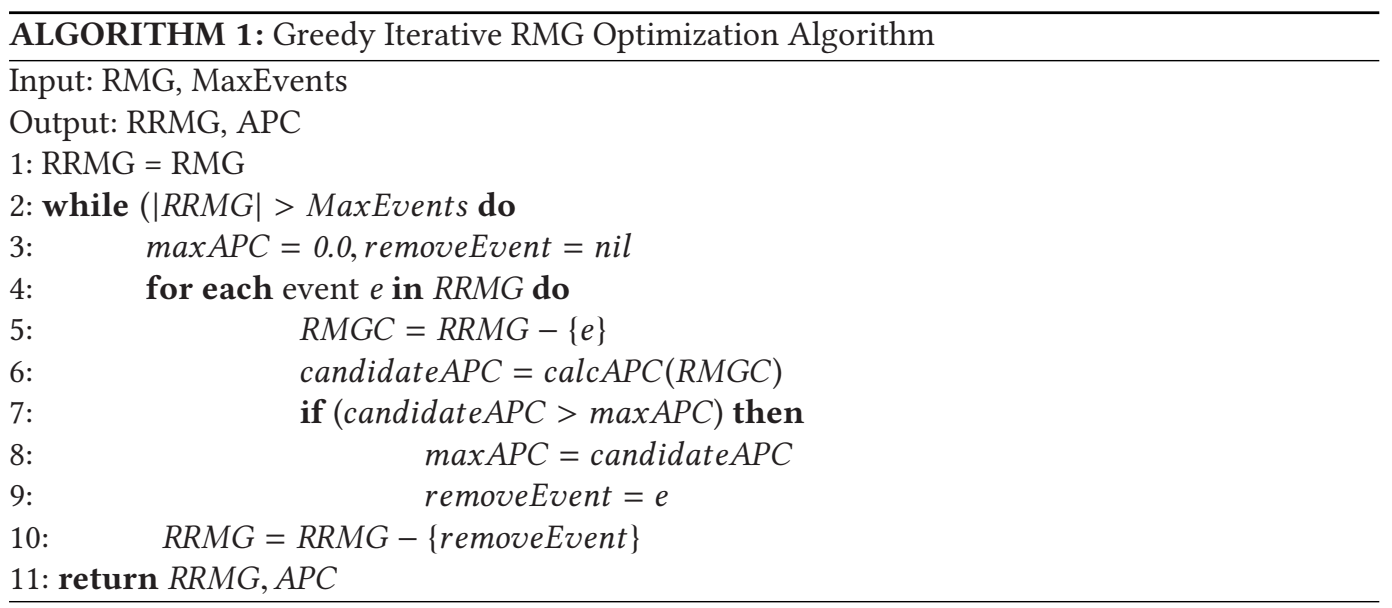

optimization algorithm. Note that while an exhaustive optimization algorithm would yield the optimal result, for the size RRMG considered in this article, determining the optional solution is infeasible, requiring searching approximately $2^{85}$ possible configurations in the case of the pacemaker. Algorithm 3 presents the simulated annealing optimization algorithm for optimizing the RMG given the maximum events constraint. The algorithm starts with a randomly generated solution. In each iteration of the algorithm, the neighboring candidate RRMG is determined by randomly replacing one event in the RRMG with a random event not in the RRMG. If the APC is higher, then the candidate RMG is selected. Otherwise, the candidate RRMG is probabilistically selected based on the simulated annealing algorithm's temperature schedule. A linearly decreasing temperature schedule is used. 

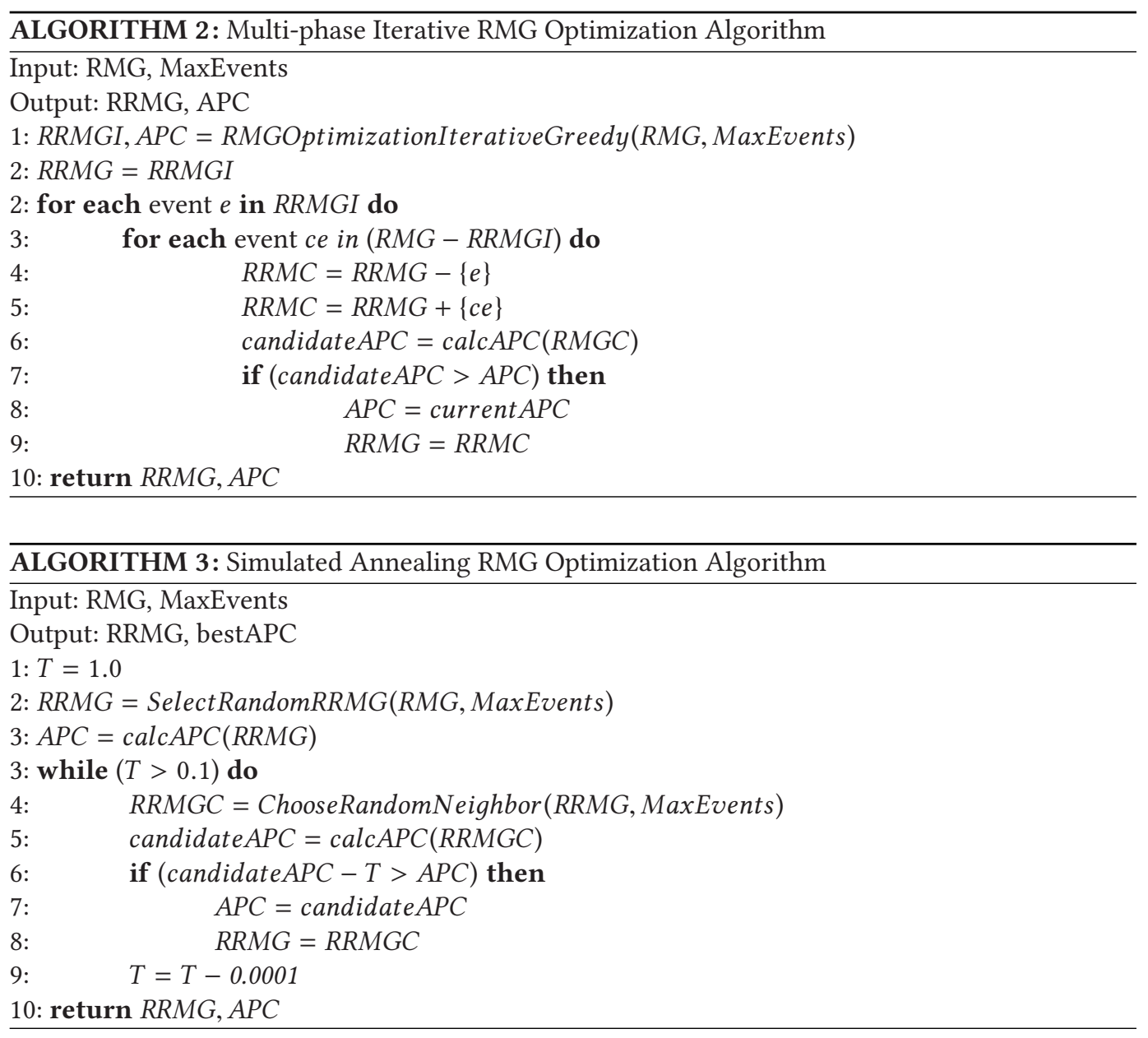

\section{RMG CONFIGURATION AND BINARY UPDATE}

The NIRM hardware is configurable, such that the RMG used to monitor the software application much be configured within the on-device start. While that configuration could be performed using a bootloader, or similar process, our current approach integrates the configuration with in the application binary itself. Thus, the application binary must be updated to incorporate the RMG configuration such that the NIRM hardware is configured when the system executes. During software compilation, a NIRM configuration stub is incorporated. The configuration stub includes the software required to program the NIRM with a placeholder configuration that programs the NIRM hardware but monitors no events. This stub is integrated and compiled with the software application. This compile-time integration ensures that the addresses of the software events can be readily determined from analyzing the application binary (i.e., the final address of the monitored won't be changed due to the insertion of the NIRM configuration process). The application binary is then analyzed to determine the addresses that correspond to each event within the RMG. This process first matches the events and messages with the UML sequence diagrams to lines of code within the software, and then matches the lines of code to specific instruction in the application binary. Thus, given the RMG and the compiled software binary, the RMG configuration 


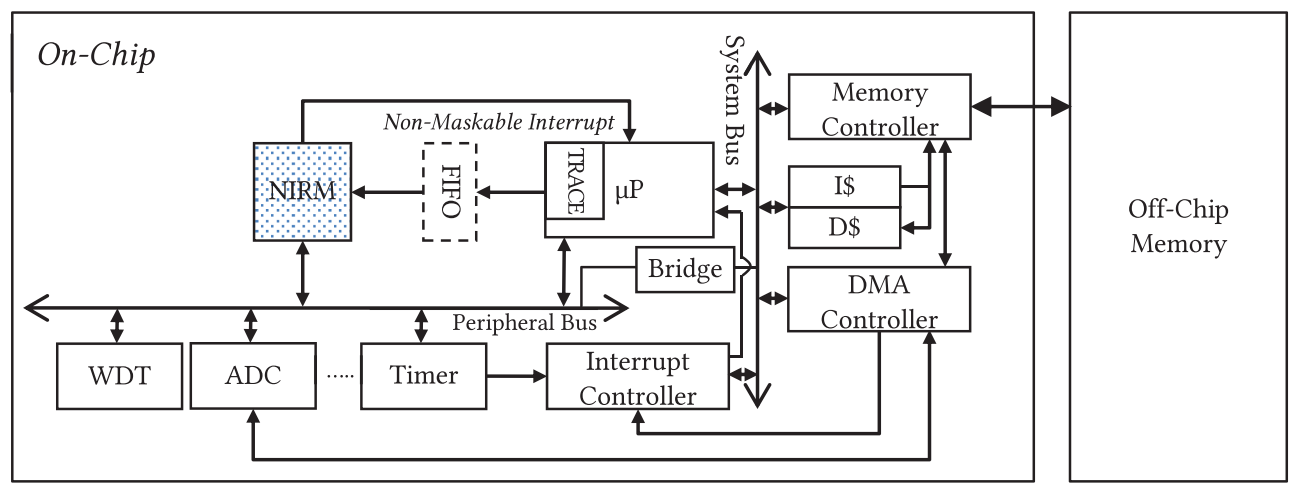

Fig. 4. NIRM hardware integrated within a typical embedded system architecture.

generation analyzes the binary to identify the addresses corresponding to each monitored event and updates the configuration stub in the application binary with the events configuration. That configuration includes the event address, event dependencies, and timing requirements. When the application execution starts, the updated configuration stub programs the NIRM hardware with the selected RMG.

\section{NIRM HARDWARE}

Figure 4 shows the integration of the NIRM hardware within a typical embedded system architecture. The NIRM hardware's main integration is to the trace port of the microprocessor on which the monitored software application executes. For systems in which the NIRM hardware does not execute at the same speed as the processor (e.g., due to a very high processor frequency or for low-power implementations), an optional FIFO can be utilized between the trace port and NIRM hardware. The FIFO would both serve to synchronize between the two clock domains as well as provide storage for a burst of monitoring events. The NIRM hardware also provides a memorymapped interface using the system, or peripheral, bus to support configuration and access to the runtime state and verification date, of which the latter is important for post-failure analysis.

Figure 5 presents a high-level overview of the NIRM hardware. The NIRM hardware is directly integrated on-chip within the SOC and interfaces with the processor trace port to non-intrusively monitor the program counter to detect system events. Integrating the NIRM on-chip and using the internal processor trace port has the advantage of not requiring any modifications to the processor core and is not restricted by bandwidth constraint of the external trace interface.

The NIRM hardware consists of the following: (a) a trace port (TracePort) interface that monitors the processor's program counter (TracePC) along with a 1-bit signal TraceValidInst indicating when a valid instruction is being executed; (b) a memory-mapped interface for configuring the NIRM hardware and post-failure analysis of system execution; (c) an RMG Configuration component that stores the RMG consisting of events' start and addresses, timing requirements, and dependency requirements; (d) an EventMonitor component for each event that detects each event's execution and measures the timing of the event execution; (e) a dependency monitor (DepMonitor) component that checks for dependency violations; and (f) a Failure analysis component that indicates if any dependency of timing failure occurred by asserting a non-maskable interrupt.

The TracePC signal represents the current PC address of the microprocessor and TraceValidInstr is a signal indicating whether the current instruction is valid. Each EventMonitor employs two comparators to measure the time that matches valid PC addresses, and it has a time monitor and 


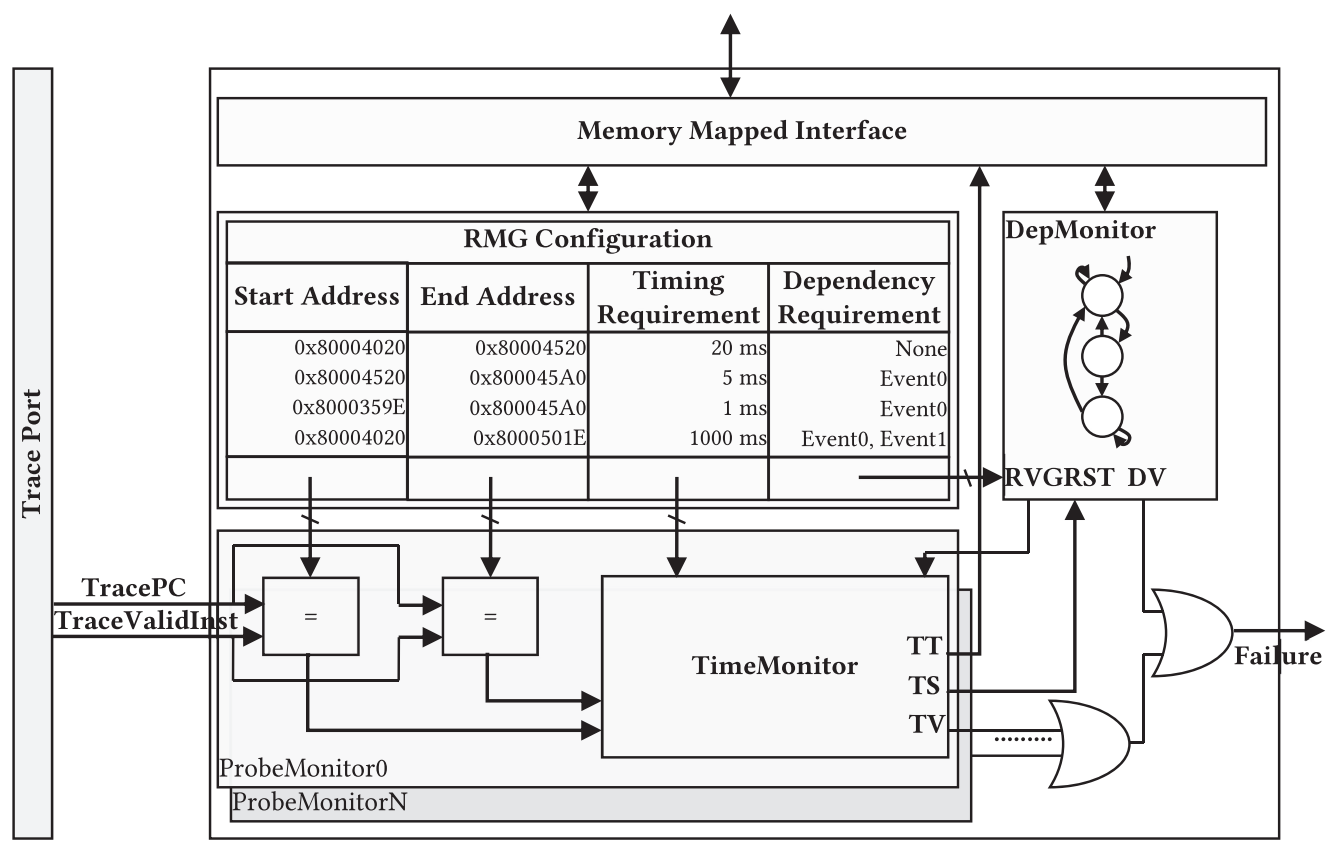

Fig. 5. Overview of NIRM hardware component that directly interfaces with the processor trace port to non-intrusively monitor the program counter to detect system events.

transmits Time Taken (TT), Time Sequence (TS), and Timing Valid (TV) signals to other units based on the information obtained from the start address, end address, and timing requirement from the configuration. The finite state machine of the dependency monitor checks the dependencies among events with the dependency requirement from the configuration.

Both timing and dependency monitors report failure by ORing timing valid (TV) and dependency valid (DV) signals. For debugging or post-failure actions, the information of these monitors can be communicated to the system designer via the memory mapped interface on request. When a failure is detected, the NIRM hardware freezes the current monitoring state until reset from the software application. This approach is used to ensure the state of events that led to the failure is maintained and can be read by the system to analyze or log the failure. The current implementation uses a non-maskable interrupt to inform the system a failure occurred. The interrupt service routine reads that failure details and immediately resets the NIRM hardware. We note that with some modification, the NIRM hardware could log the failure in local storage, such that the monitoring does not block until the failure is read, but we leave this implementation as future work.

Note that NIRM hardware does not require any modification of processors. Modern microprocessors in SOC design stage allow us to utilize trace port signals for the purpose of performance monitoring.

\section{EXPERIMENTAL RESULTS}

We developed an FPGA-based prototype for the NIRM hardware and two embedded applications modeling an autonomous vehicle and a smart connected pacemaker. The system includes a $150 \mathrm{MHz}$ MicroBlaze, AXI bus, $32 \mathrm{~Kb}$ instruction cache, $32 \mathrm{~Kb}$ data cache, Ethernet controller, and several standard peripherals (e.g., timer, UARTs). The NIRM hardware integrated on-chip 
Table 1. NIRM Hardware Area Reported in Lookup Tables (LUTs), Flip-Flops (FFs), and Power Overhead for Various Number of RMG Events

\begin{tabular}{|c|r|r|c|}
\hline Events & LUTs & \multicolumn{1}{c|}{ FFs } & Power Overhead \\
\hline \hline 8 & 708 & 526 & $0.17 \%$ \\
\hline 16 & 1428 & 1052 & $0.27 \%$ \\
\hline 32 & 2850 & 2104 & $0.85 \%$ \\
\hline 64 & 5697 & 4192 & $2.86 \%$ \\
\hline 128 & 11611 & 7451 & $10.69 \%$ \\
\hline
\end{tabular}

supports between 32 and 128 events. The system was synthesized for a Xilinx Virtex-6 FPGA (xc6vlx240t).

The NIRM hardware supports a maximum frequency of $274 \mathrm{MHz}$ for the targeted FPGA. While this frequency is high enough to monitor the MicroBlaze processor at its maximum frequency of $250 \mathrm{MHz}$, we implemented the system using a $150 \mathrm{MHz}$ for the processor, NIRM hardware, and system bus, which corresponds to the maximum system bus frequency for the targeted FPGA. We reiterate that for systems in which the processor runs at a higher frequency or where the processor and system bus frequencies are different, a FIFO (Lee and Lysecky 2015; Nair and Lysecky 2008) between the processor trace port and NIRM hardware can be used, although we do not evaluate such implementation here. Last, we note that we expect the NIRM hardware frequency to scale with processor frequencies when using alternative process technologies, such as ASIC or full custom.

Table 1 presents the NIRM hardware's area, reported in lookup tables (LUTs) and flip-flops (FF), and power overhead for various numbers of supported RMG events. The area overhead ranges from 1.9 to $22.6 \%$ relative to the small base system without the NIRM hardware. The area used by the NIRM utilizes 0.3 to $5.1 \%$ of the total available FPGA area. Furthermore, the NIRM hardware's area requirements scale linearly with the number RMG events. The power overhead is reported as the percentage overhead in total power consumption compared to a base MicroBlaze system without the NIRM hardware. The power overhead ranges from less than 1 to $10.7 \%$ for depending on the size of the NIRM hardware compared to the base power, $3436 \mathrm{~mW}$. We note that NIRM implementation did not utilize any low-power design techniques, and the power overhead could be further reduced. One simple approach to reduce the power would be to operate the NIRM hardware at a much lower frequency. On average, the occurrence of events is significantly lower than the one event per cycle, so the NRIM hardware could operate at much lower internal frequencies, thereby reducing power consumption. However, we leave the power optimization of the NIRM hardware as future work.

\subsection{Embedded Applications}

We use two representative embedded applications to evaluate the NIRM approach, namely an autonomous vehicle and a smart connected pacemaker. Each embedded application was implemented as a multi-tasked application using Xilinx xilkernel 5.00a.

The first embedded application is a simplified prototype of the integrate of an autonomous vehicle's navigation, speed, and steering control subsystems. Figure 6 presents a UML sequence diagram for these subsystems and corresponding RMG automatically extracted from the sequence diagram. The autonomous vehicle's operations consist of two high-level tasks: (1) acquiring data from various input sensors, which are represented by the sequence of events A to B, and (2) processing sensor data, making navigation decisions, and controlling the vehicle's actuators, which 


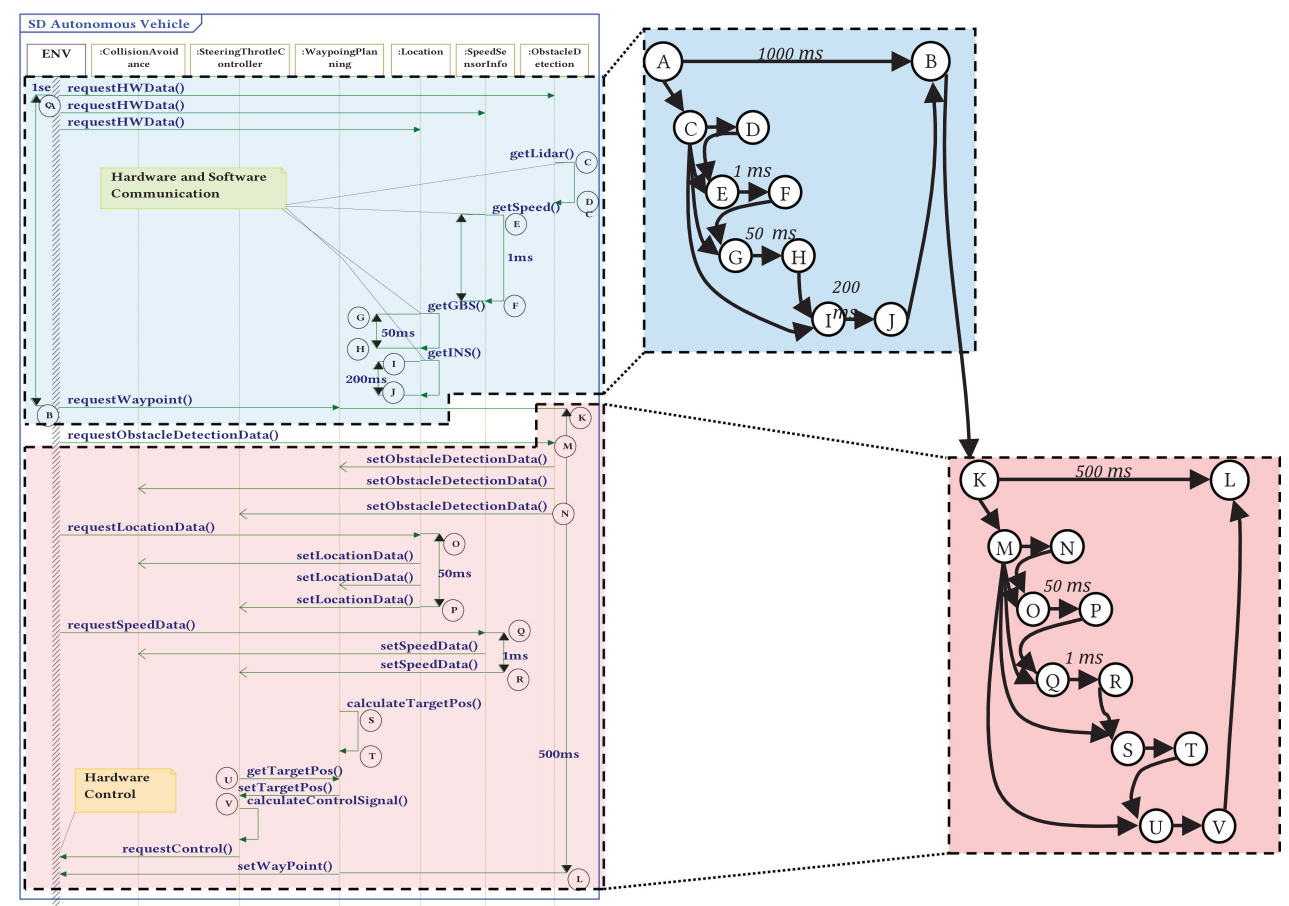

(a)

(b)

Fig. 6. (a) Example UML sequence diagram for autonomous vehicle and (b) corresponding RMG extracted from the sequence diagram.

are represented by the sequence of events $\mathrm{K}$ to $\mathrm{L}$. The autonomous vehicle's RMG consists of 22 events, 30 dependencies, and 7 timing requirements.

The second embedded application is a smart connected pacemaker supporting physicianconfigurable pacing configurations, communication of cardiac activity to a home monitoring device, and emergency physician notification for significant cardiac events. From a high-level point of view, the smart connected pacemaker consists of record atrial interrupt, AVI interrupt, record ventricle interrupt, VAI interrupt, cardiac activity analysis and logging, physician warning transmission, and daily cardiac activity communication components. Figure 7 presents a high-level view of the smart connected pacemaker's sequence diagrams (note that low-level details are omitted due to the complexity of the system requirements) and corresponding RMGs represented in Figure 8. The smart connected pacemaker's RMG consists of 100 events, 101 dependencies, and 53 timing requirements.

\subsection{Analysis of Detection Rate}

To evaluate the effectiveness of the NIRM approach to verify execution and detect potential failures at runtime, we consider several common failure cases, summarized in Table 2, including timing failures (Macaulay 1992), dependency failures (Leveson and Turner 1993), synchronization failures (Jones 1997), and sensor failures (Bonakdarpour et al. 2011). For each failure type, we inserted the modeled errors within each system at numerous locations. For each RRMG and each failure type, we executed the system 100 times, randomly activating one error during each execution, and measuring the detection rate achieved by the NIRM approach. 

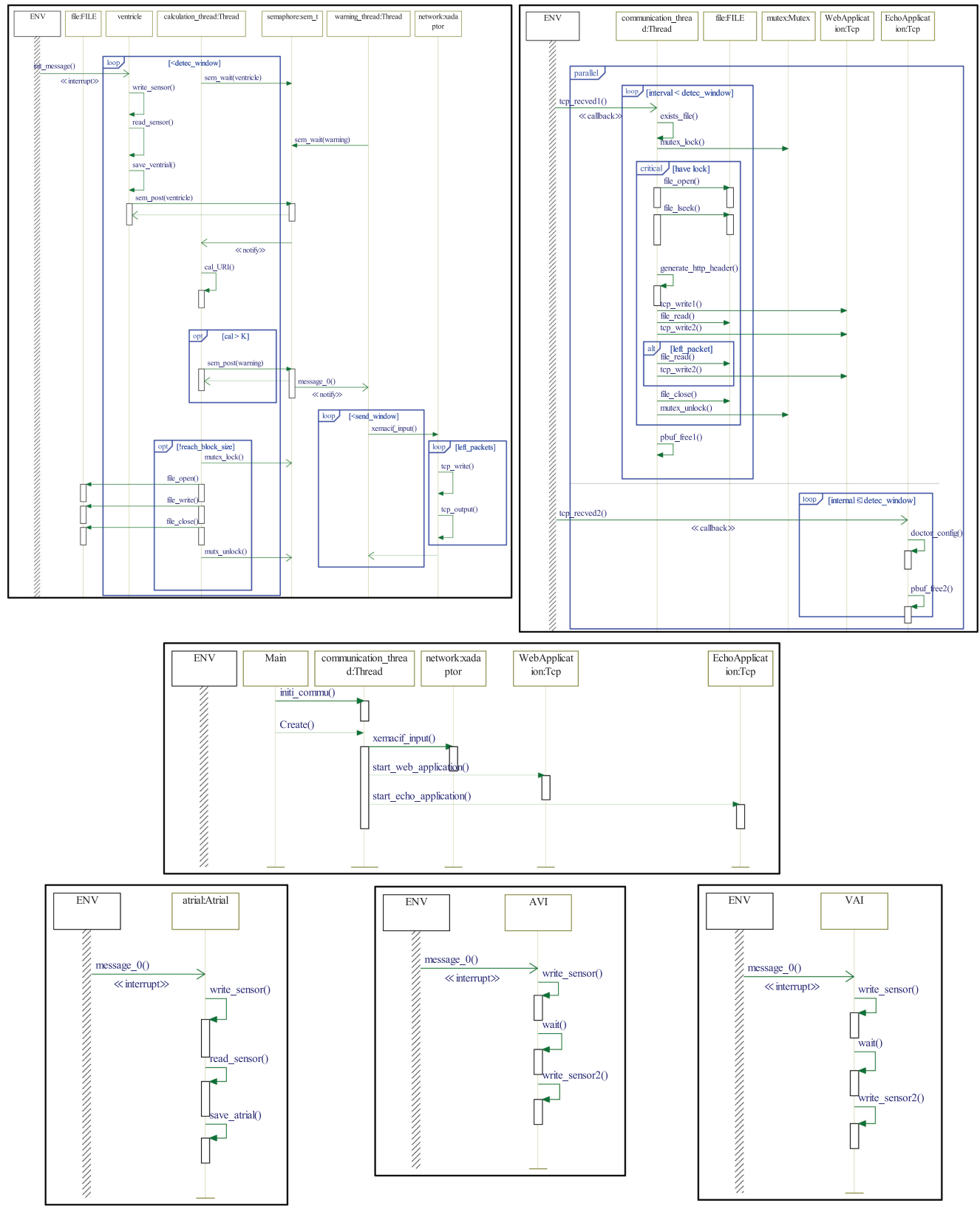

Fig. 7. UML sequence diagrams for smart connected pacemaker. Note: These diagrams are intended to highlight the characteristic and complexity of the system, so some details may not be visible.

While the NIRM hardware is programmable, the number of events monitored at runtime will be fixed at design time when the system is implemented. Understanding the tradeoffs between monitoring coverage, detection rates for common failures, and hardware resources requirements is needed to assist designers in determining the size of the NIRM hardware. To evaluate this, we 


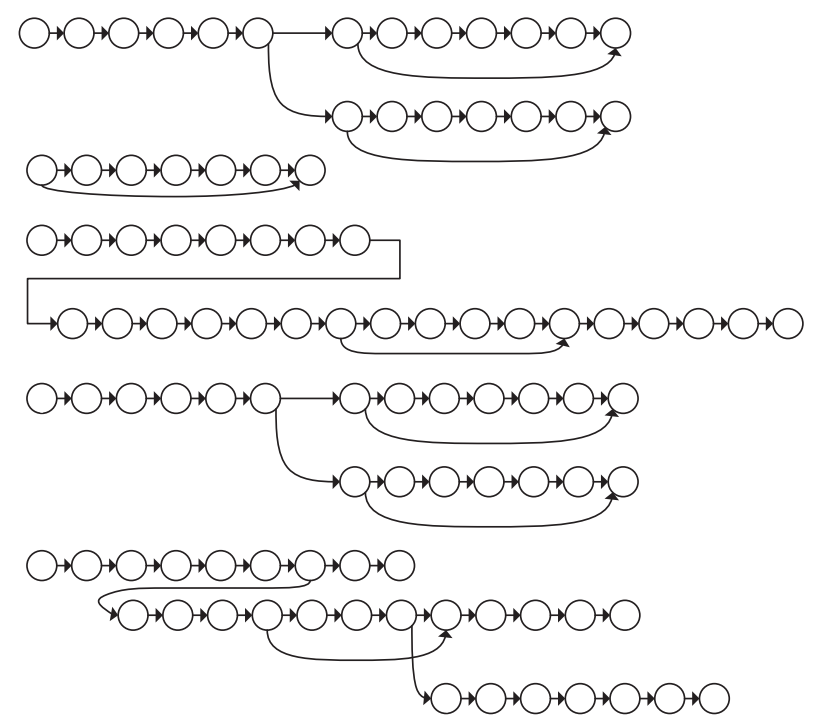

Fig. 8. Corresponding RMG extracted from the sequence diagram in pacemaker example. Note: Event IDs and timing requirements are omitted due to the complexity of the RMG.

Table 2. Summary of Failure Types and Corresponding Failure Injection Method

\begin{tabular}{|c|c|c|}
\hline Failure & Description & Failure Injection Method \\
\hline Timing Failure & $\begin{array}{c}\text { Task execution takes longer } \\
\text { than the WCET for that task, } \\
\text { leading to violation(s) of } \\
\text { real-time constraints }\end{array}$ & $\begin{array}{c}\text { Insert timing delays ranging } \\
\text { from } 500 \mu \text { s to 1ms within } \\
\text { targeted computational tasks. }\end{array}$ \\
\hline $\begin{array}{c}\text { Dependency } \\
\text { Failure }\end{array}$ & $\begin{array}{c}\text { Operations with implicit } \\
\text { dependencies are executed out } \\
\text { of order (e.g., scheduling errors, } \\
\text { delays from blocking IO) }\end{array}$ & $\begin{array}{c}\text { Randomize execution order of } \\
\text { dependent operations. }\end{array}$ \\
\hline $\begin{array}{c}\text { Synchronization } \\
\text { Failure }\end{array}$ & $\begin{array}{c}\text { Mutex synchronization failures } \\
\text { can often lead to system } \\
\text { deadlocks. }\end{array}$ & $\begin{array}{c}\text { Randomize mutex lock/unlock } \\
\text { failures. }\end{array}$ \\
\hline Sensor Failure & $\begin{array}{c}\text { Sensor failures resulting in } \\
\text { incorrect data causing incorrect } \\
\text { control decisions. }\end{array}$ & $\begin{array}{c}\text { Simulate capture of erroneous } \\
\text { sensor data and resulting } \\
\text { processing of erroneous data as } \\
\text { if it were valid. }\end{array}$ \\
\hline
\end{tabular}

consider several RMGs for the autonomous vehicle system with events constraints ranging from the full RMG with 15 events to an RRMG with only 5 events. For the smart connected pacemaker system, we similarly evaluate the detection rate for various RMG sizes ranging from the full RMG with 100 events to an RRMG with 14 events.

Figure 9 presents the resulting error detection rate for the autonomous vehicle system, for each error type and for differing constraints on the number of events within the RRMG, using the iterative greedy RMG optimization algorithm. The NIRM approach achieves a $100 \%$ detection rate for all error types when utilizing the full RMG. Additionally, the approach achieves a detection 


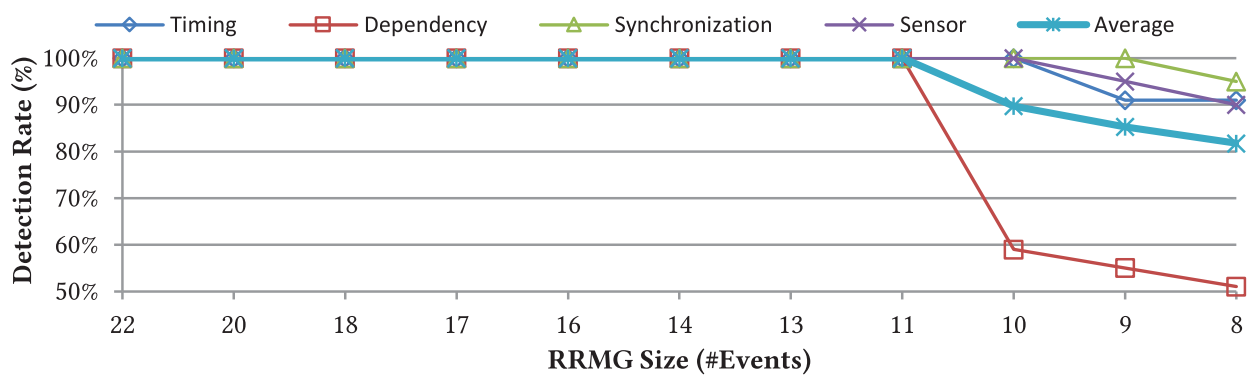

Fig. 9. Detection rate for timing failures, dependency failures, synchronization failures, sensor failures, and overall average detection rate for the autonomous vehicle system.

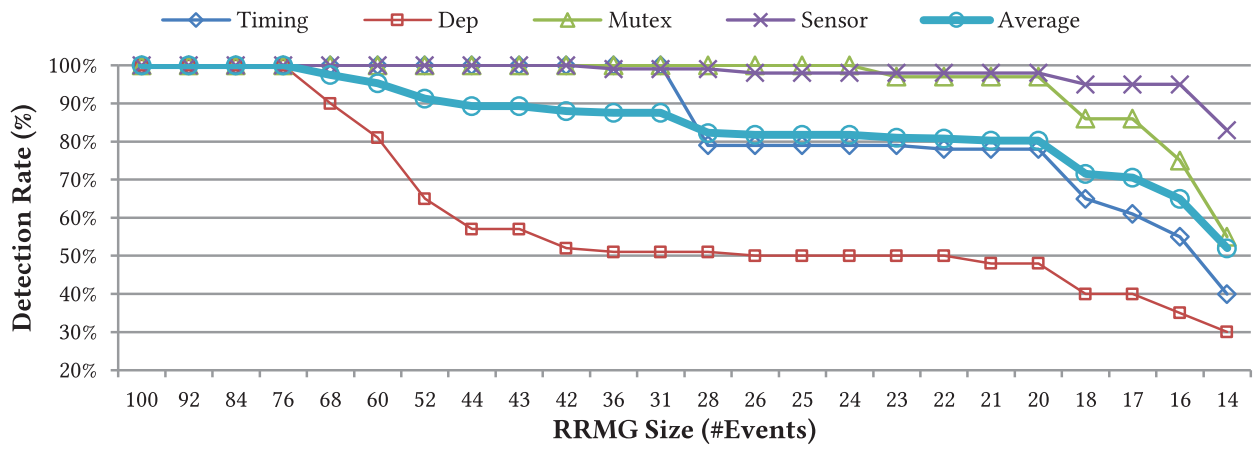

Fig. 10. Detection rate for timing failures, dependency failures, synchronization failures, sensor failures, and overall average detection rate for smart connected pacemaker.

rate greater than $90 \%$ with only 10 events for timing, synchronization, and sensor failures. As the number of events decreases, the approach performs best for synchronization failures, achieving a $100 \%$ detection rate with 9 events. This is due to that fact that synchronization failures can be detected both from the sequence and timing requirements. For an RRMG with 10 events, the detection rate for dependency failures drops to less than $60 \%$. Notably, the ESC for the resulting RRMG is $66 \%$, which is relatively close to the detection rate, indicating ESC is a good indicator of dependency failures. Furthermore, the APC for an RRMG with 8 events is $80 \%$, which is also close to the average detection rate across all failure types of $90 \%$.

Figure 10 presents the error detection rate for the smart connected pacemaker system, for each error type and for differing constraints on the number of events within the RRMG, again using the iterative greedy RMG optimization algorithm. NIRM achieved a $100 \%$ detection rate for all failure cases when using at least 76 events. As the number of events in the RRMG decreases, NIRM performs best at detecting timing, sensor, and mutex failures and performs worst for dependency failures. With 31 events, the detection rates for timing, mutex, and sensor failures is 100, 100, and $99 \%$, whereas the detection rate for dependency failures is $51 \%$. With 20 events, which is only $20 \%$ of the RMG, the average detection rate is $80.3 \%$.

We further analyzed the performance of the four RMG optimization algorithms, including the greedy iterative (GI), multi-phase iterative (MI), simulated annealing (SA), and exhaustive (EX), where feasible. Figure 11(a) and (b) presents the APC and average detection rates for all failures types for the autonomous vehicle system. Note that for the autonomous vehicle system's RMG, exhaustive optimization is feasible, but for the smart connected pacemaker's RMG, exhaustive 


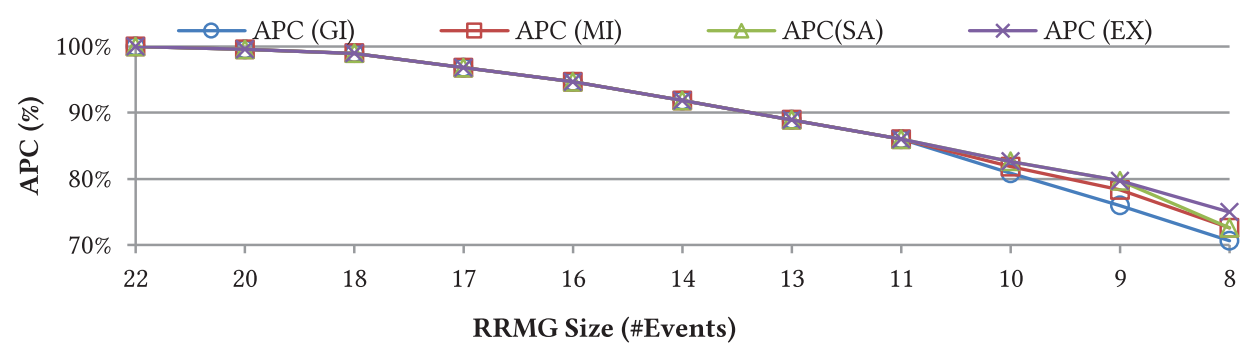

(a)

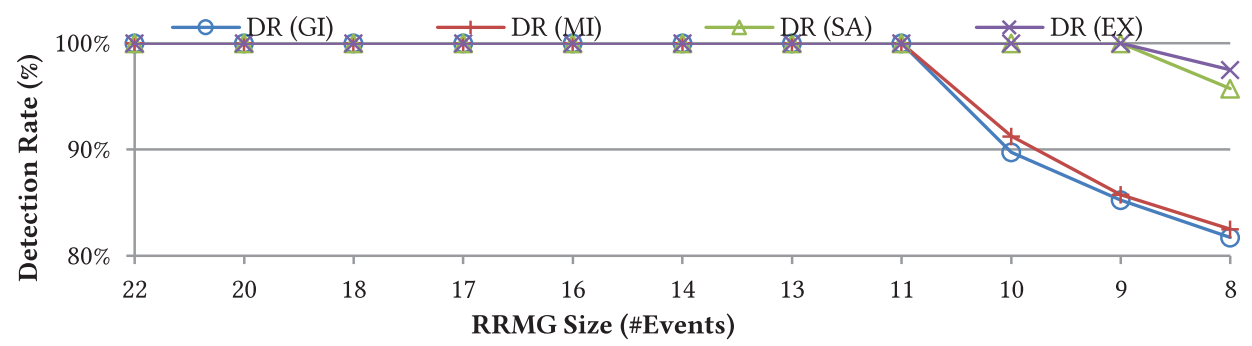

(b)

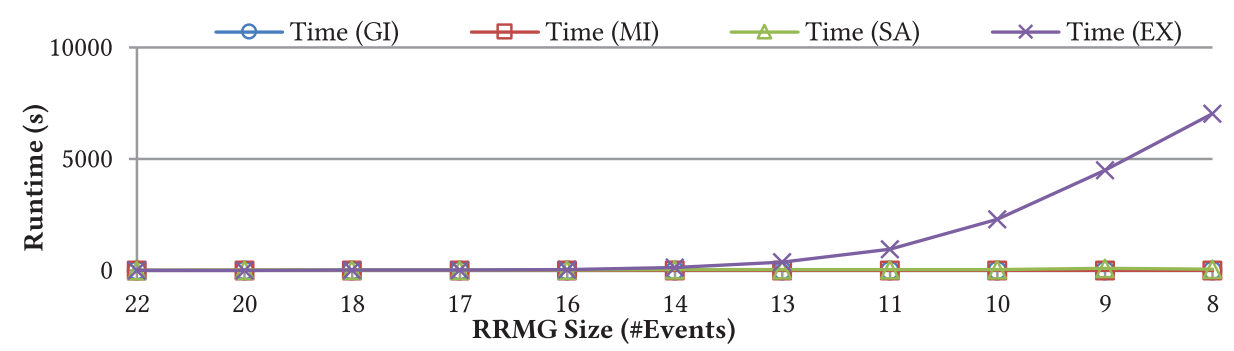

(c)

Fig. 11. (a) Average path coverage (APC), (b) detection rate (DR), and (c) execution runtime for greedy iterative (GI), multi-phase iterative (MI), simulated annealing (SA), and exhaustive (EX) RMG optimization algorithms for the autonomous vehicle system.

optimization is not feasible due to the system complexity. Figure 11(a) shows a similar decreasing trend in APC for all four optimization algorithms with only small differences between resulting APC. For example, for the smallest constraint, the difference in APC between EX and GI, which have the best and worst APC, is only $2.34 \%$. However, by examining the actual detection rate (Figure 11(b)), a much larger difference can be observed. The EX and SA algorithms outperform the MI and GI algorithms when the RRMG size decreases below 8 events. With just 8 events, EX achieves a $97.5 \%$ detection rate, and SA achieves a $95.8 \%$ detection rate. This clearly shows the tradeoff in optimization quality that comes with the fast, iterative algorithms. MI achieves an $82.5 \%$ detection rate, which is $15.3 \%$ lower than EX. Additionally, the multi-phase optimization approach of $\mathrm{MI}$ achieves a $0.75 \%$ improvement in the detection rate compared to GI. Note that the ordering of algorithm performance from best to worst was EX, SA, MI, and GI, as measured both by the $\mathrm{APC}$ and by the detection rate.

Figure 11(c) presents the runtime for each optimization algorithm. As expected, the exclusive optimization algorithm has the longest runtime. For an RMG of just 22 events, the EX algorithm requires more than $1 \mathrm{~h}$ to find an optimized RRMG with half the number of events, and $2 \mathrm{~h}$ to find an optimized RRMG for the smallest constraint of 8 events. The SA algorithm requires only 260s 


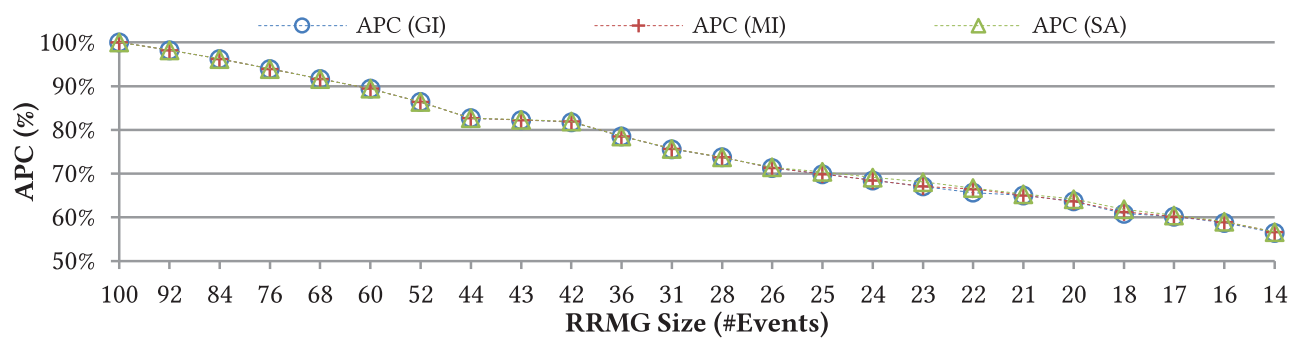

(a)

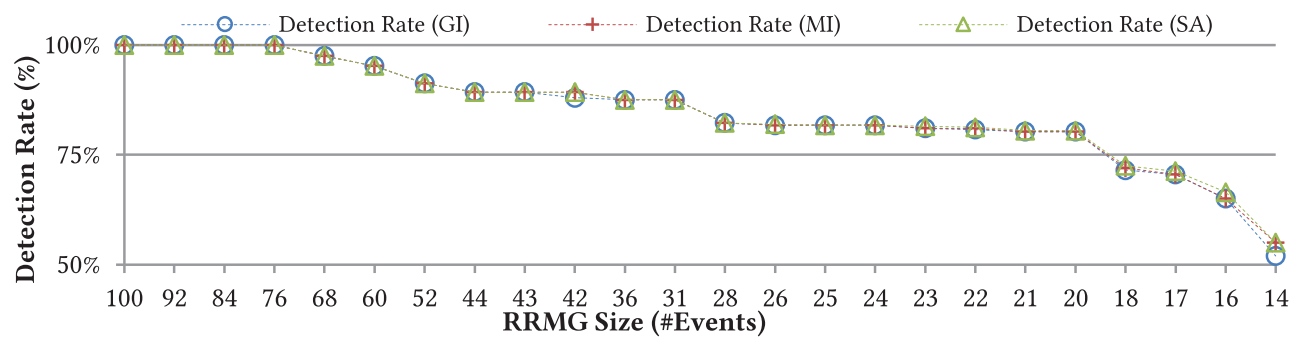

(b)

Fig. 12. (a) Average path coverage (APC) and (b) detection rate (DR for greedy iterative (GI), multi-phase iterative (MI), and simulated annealing (SA) RMG optimization algorithms for the smart connected pacemaker.

to find an optimized RRMG for a constraint of 8 events. In contrast, the GI and MI algorithms are much faster, as expected. The GI and MI algorithms require only $0.56 \mathrm{~s}$ and $1.68 \mathrm{~s}$, respectively, to find the optimized RRMG. The MI algorithm is $6281 \times$ and $36 \times$ faster than the EX and SA algorithms, respectively.

For the smart connected pacemaker, we analyzed the performance of the greedy iterative (GI), multi-phase iterative (MI), and simulated annealing (SA) optimization algorithms. Figure 12(a) and (b) presents the APC and average detection rates for all failures types for the smart connected pacemaker. For this benchmark, the EX algorithm was not evaluated as it would require $3.8 \times$ $10^{25}\left(\sum_{n=1}^{100} 100\right.$ choose $\left.n\right)$ APC evaluations, which yields infeasible runtime. While on average the SA algorithm achieves the highest APC and detection rate, the difference between SA and the MI and GI algorithm is very small, with an average difference of only $0.45 \%$. To further demonstrate, Table 3 presents the increase in APC and detection rate for the SA and MI algorithms compared to the GI algorithm for any RRMG event constraint that yielded an increase in either metric. For 14 of the constraints considered, the SA algorithm achieves a higher detection rate than GI, with an average and maximum increase of 0.48 and $2.3 \%$, respectively. The MI algorithm's refinement phase is able to improve on the GI algorithm for 7 of the event constraints, yielding improvements in the detection rate of up to $1.2 \%$.

As shown in Figure 13, the execution time for the SA algorithm ranges from 21 minutes to 108 minutes, while MI requires less than 7s. Further, the GI algorithm requires less than $1 \mathrm{~s}$ for any constraint on the RRMG size. Overall, the MI RRMG optimization algorithm provides an excellent tradeoff of $925 \times$ speedup with a reduction in the detection rate of less than $2.3 \%$.

\subsection{Detection Latency and Post-Failure Analysis}

While APC and detection rate help to evaluate how many failures the NIRM can detect for a given RRMG, the detection latency and ability to perform post-failure analysis are also important. We 
Table 3. APC and Detection Rate Increase Percentage for MI and SA Optimization Compared to GI Optimization

\begin{tabular}{|c|c|c|c|c|}
\hline \multirow{2}{*}{ Events } & \multicolumn{2}{|c|}{ MI } & \multicolumn{2}{c|}{ SA } \\
\cline { 2 - 5 } & $\begin{array}{c}\text { APC } \\
\text { Increase }\end{array}$ & $\begin{array}{c}\text { Detection Rate } \\
\text { Increase }\end{array}$ & $\begin{array}{c}\text { APC } \\
\text { Increase }\end{array}$ & $\begin{array}{c}\text { Detection Rate } \\
\text { Increase }\end{array}$ \\
\hline 43 & - & - & $0.11 \%$ & $0.00 \%$ \\
\hline 42 & $0.20 \%$ & $1.42 \%$ & $0.20 \%$ & $1.42 \%$ \\
\hline 31 & $0.14 \%$ & $0.00 \%$ & $0.14 \%$ & $0.00 \%$ \\
\hline 26 & - & - & $0.21 \%$ & $0.31 \%$ \\
\hline 25 & - & - & $0.73 \%$ & $0.00 \%$ \\
\hline 24 & - & - & $0.97 \%$ & $0.00 \%$ \\
\hline 23 & - & - & $1.53 \%$ & $0.62 \%$ \\
\hline 22 & $1.19 \%$ & $0.31 \%$ & $1.56 \%$ & $0.62 \%$ \\
\hline 21 & - & - & $0.38 \%$ & $0.31 \%$ \\
\hline 20 & - & - & $0.81 \%$ & $0.31 \%$ \\
\hline 18 & $0.69 \%$ & $0.70 \%$ & $1.69 \%$ & $1.40 \%$ \\
\hline 17 & - & - & $0.41 \%$ & $1.06 \%$ \\
\hline 16 & $0.35 \%$ & $0.00 \%$ & $0.51 \%$ & $2.31 \%$ \\
\hline 14 & $0.36 \%$ & $5.77 \%$ & $0.36 \%$ & $5.77 \%$ \\
\hline
\end{tabular}

Only the event constraints that yielded an increase are shown.

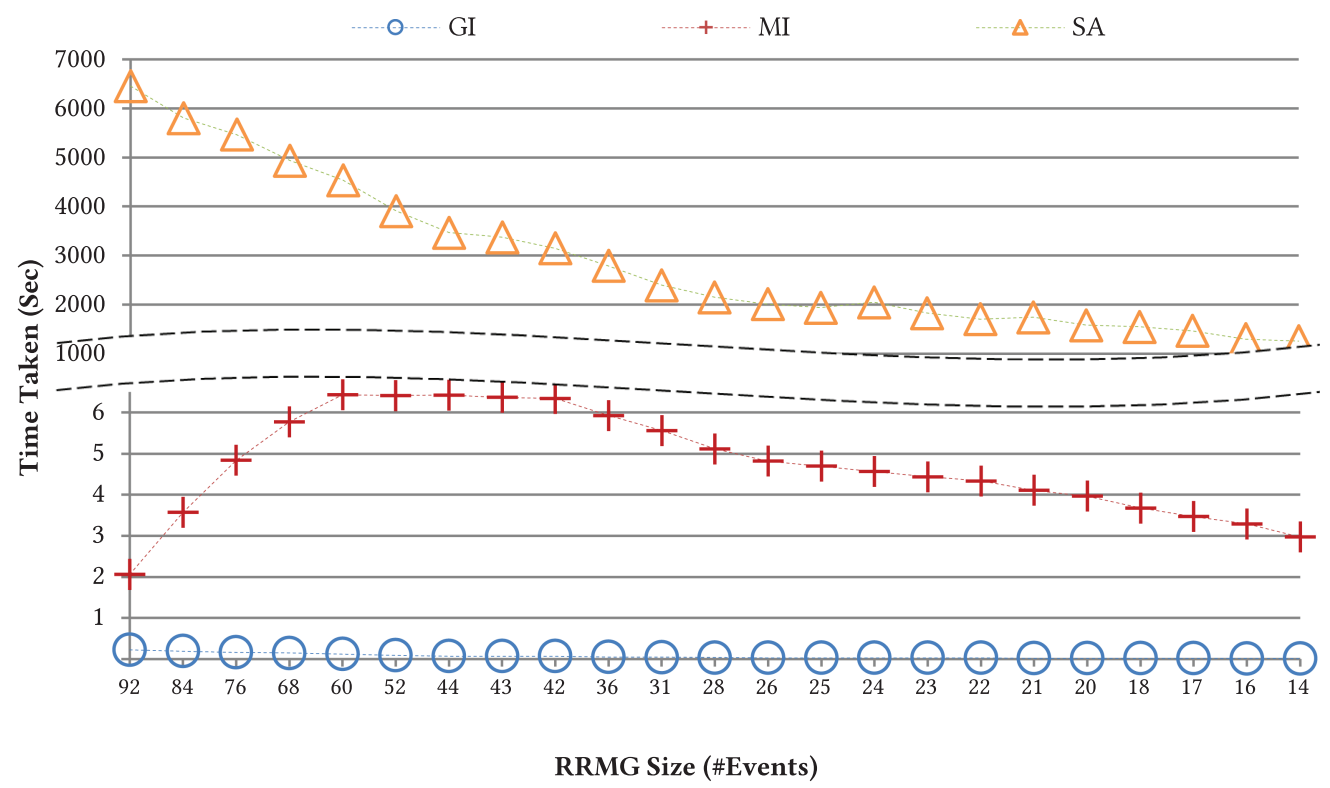

Fig. 13. Execution runtime for GI, MI, and SA optimization algorithms for the smart connected pacemaker.

define the detection latency as the time between the occurrence of the error and asserting the alert indicating a requirement violation occurred.

Table 4 presents the detection rate and detection latency for five different RRMGs for the autonomous vehicle system considering the mutex failure. Notably, all of these RRMGs have the same 
Table 4. RRMG Size, Detection Rate, and Detection Latency (in Cycles) for Various RRMGs for the Autonomous Vehicle System Considering the Mutex Failure Case

\begin{tabular}{|c|c|c|}
\hline RRMG Size(events) & Detection Rate & Detection Latency (cycles) \\
\hline \hline 15 & $100 \%$ & 19125112 \\
\hline 14 & $100 \%$ & 19125112 \\
\hline 13 & $100 \%$ & 19929817 \\
\hline 12 & $100 \%$ & 20134119 \\
\hline 11 & $100 \%$ & 25134119 \\
\hline
\end{tabular}

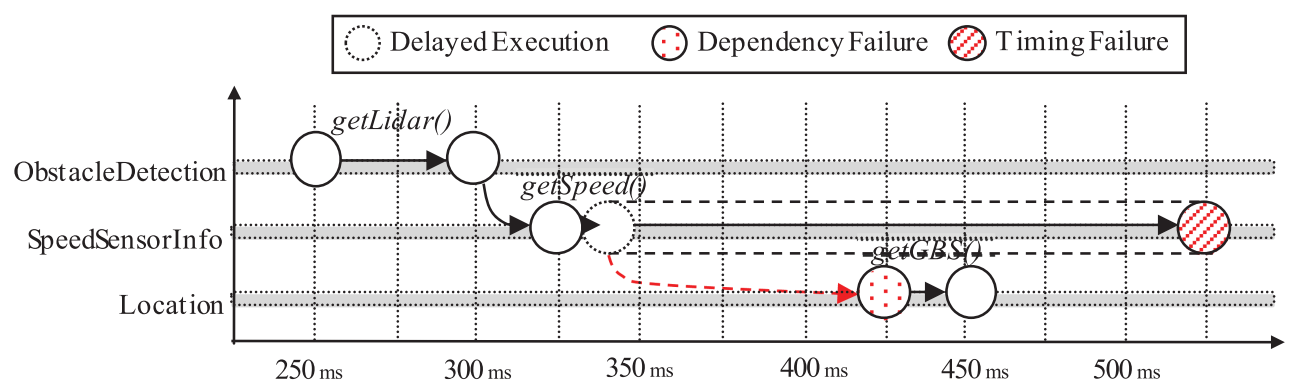

Fig. 14. An example of failure backtracking for post-failure analysis in the autonomous vehicle system.

detection rate but yield different detection latencies. Generally, an RRMG with more events will yield a lower detection latency. For example, consider a sequence of executions dependencies of $\mathrm{A}, \mathrm{B}$, and $\mathrm{C}$. If $\mathrm{A}$ fails to occur before $\mathrm{B}$ and $\mathrm{C}$, then a dependency violation will be detected. An RRMG with events $A, B$, and $C$ would detect the violation as soon as $B$ is detected. But for a smaller RRMG with just $A$ and $C$, the violation is not detected until $C$ occurs. With decreasing RRMGs size from 15 to 11 events, the detection latency increases by $31 \%$.

The runtime execution data collected by the NIRM hardware can be used for post-failure analysis. Specifically, NIRM enables failure backtracking that enables a designer (or a failure mitigation process) to analyze the sequence of events that resulted in the requirements violation. The NIRM hardware maintains data on each events execution, including the execution time of events. The RRMG configured within the NIRM hardware also keeps track of the expected dependencies and sequences of events. Based on this information, one can trace backward to determine the sequence and timing of events that occurred. Figure 14 shows an example of failure backtracking for a timing failure within the autonomous vehicle system. The function getSpeed() has a prerequisite to execute getLidar(), while the getGBS() function has the getSpeed() function as a prerequisite. All of the above functions are performed within independent threads, and the software is written to perform proper synchronization so that the appropriate dependencies are executed in advance. In this example, the speed sensor is operating abnormally and the execution of the getSpeed() function takes much longer than the expected time (1ms), which leads to the get $G B S()$ function being executed before getSpeed(). In this situation, a dependency failure first occurs, followed by a timing failure of $\operatorname{getSpeed}()$ function itself. The failure backtracking can be utilized to quickly determine the root.

\subsection{Comparison with Software-Based Requirements Monitoring}

While for many embedded systems, a non-intrusive monitoring and analysis method is needed, for systems that can tolerate some overhead, a software implementation of the NIRM approach may 
Table 5. Performance Overheads of Software-based Requirement Monitoring Implementation

\begin{tabular}{|c|c|c|c|c|c|}
\hline & IRV_setEvent & IRV_setHandler & IRV_start & IRV_end & Sum \\
\hline \hline Best case & $0.02 \%$ & $0.01 \%$ & $1.80 \%$ & $2.0 \%$ & $3.83 \%$ \\
\hline Worst case & $0.19 \%$ & $0.01 \%$ & $14.08 \%$ & $24.77 \%$ & $39.05 \%$ \\
\hline
\end{tabular}

Table 6. Code Size Overheads Comparison with Software-based Requirement Monitoring

\begin{tabular}{|c|c|c|c|}
\hline & System & $\begin{array}{c}\text { Code Size } \\
\text { (bytes) }\end{array}$ & $\begin{array}{c}\text { Code Size } \\
\text { Overhead (\%) }\end{array}$ \\
\hline \multirow{2}{*}{ Base Software } & 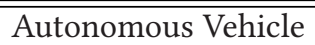 & $1720996(100 \%)$ & $0 \%$ \\
\hline & $\begin{array}{l}\text { Smart Connected } \\
\text { Pacemaker }\end{array}$ & $2605278(100 \%)$ & $0 \%$ \\
\hline \multirow{2}{*}{$\begin{array}{c}\text { Software with } \\
\text { SRM }\end{array}$} & Autonomous Vehicle & $2093517(122 \%)$ & $22 \%$ \\
\hline & Pacemaker & 2977799 (114\%) & $14 \%$ \\
\hline
\end{tabular}

be feasible. A software implementation has the advantages of not requiring additional hardware but comes at the tradeoff of execution overheads and a longer detection latency.

To understand and evaluate the overhead of a software-based NIRM implementation, we implemented Software-based Requirement Monitoring (SRM). Software instrumentation is utilized to monitor the execution of each event, specifically inserting function calls at the corresponding location within the software binary to detect each event. Each time an event is executed, the software performs the same analysis operations as the NIRM hardware, detecting events, starting and stopping software timers, and verifying sequence and timing requirements within the RRMG. The main software within the NIRM implementation are IRV_setEvent(), IRV_setHandler(), IRV_start(), and IRV_end(). The SRM incurs a 3.8\% performance overhead in the best case (with a cache hit rate of $100 \%$ ) and $39.1 \%$ performance overhead in the worst case (with caches disabled), as shown in Table 5. Note that the performance overhead will vary depending on the number of events within the RRMG, as the $I R V_{-}$start() and $I R V_{-}$end() functions are executed each time each event occurs.

Given the data collected, the performance overhead $(P)$ of the SRM can be estimated as a function of the cache hit rate $(H)$ as:

$$
P=(100+3.8) *(H)+(100+39.05) *(1-H) .
$$

For example, with a cache hit rate of $90 \%$, the SRM's performance overhead is calculated as:

$$
P=(100+3.8) *(0.9)+(100+39.05) *(0.1)=93.42+13.905
$$

$P=107.325 \approx 7 \%$ overhead.Code size overhead is also an important factor of overall system performance. Table 6 presents the code size in bytes and the percentage overhead for both the base software implementation and the instrumented software using the SRM. On average, the SRM incurs an $18 \%$ code size overhead.

We further evaluated the energy overhead of the SRM approach compared to the hardwarebased NIRM implementation. The SRM approach incurs a $5 \%$ energy overhead $(0.84 \mathrm{~mW} \cdot \mathrm{S})$, which is mainly attributed to the performance impact of the instrumentation, which is higher than the $0.26 \%$ energy overhead $(0.04 \mathrm{~mW} \cdot S)$ for the NIRM hardware.

Finally, the impact on detection latency of the SRM approach is considered. Figure 15 shows two example timelines for detecting and reporting a requirements violation for the hardware-based NIRM and software-based SRM, specifically for the autonomous vehicle system. The NIRM hardware approach requires only one cycle for a failure detection and two cycles to enter the interrupt 

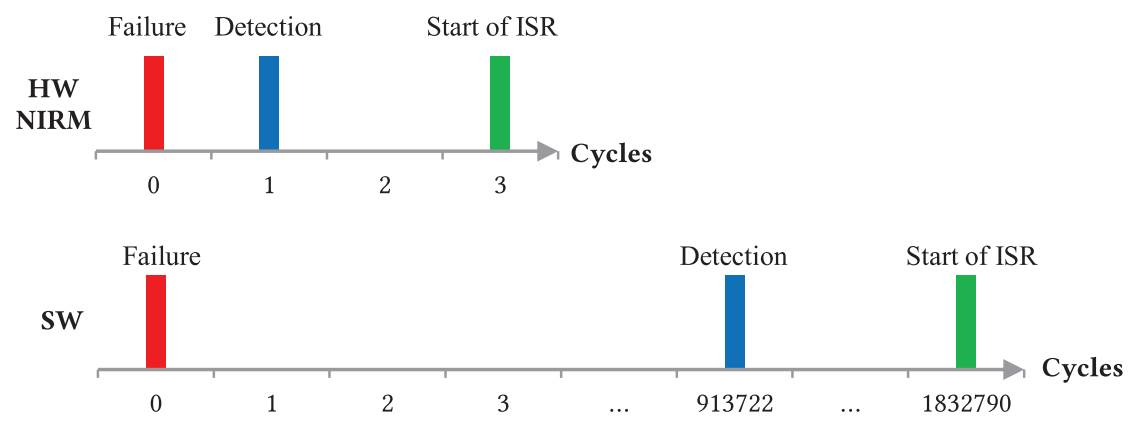

Fig. 15. Example timelines for NIRM and SRM highlighting the detection latency.

S: Start, G: Goal, TF: Timing failure, DF: Dependency Failure

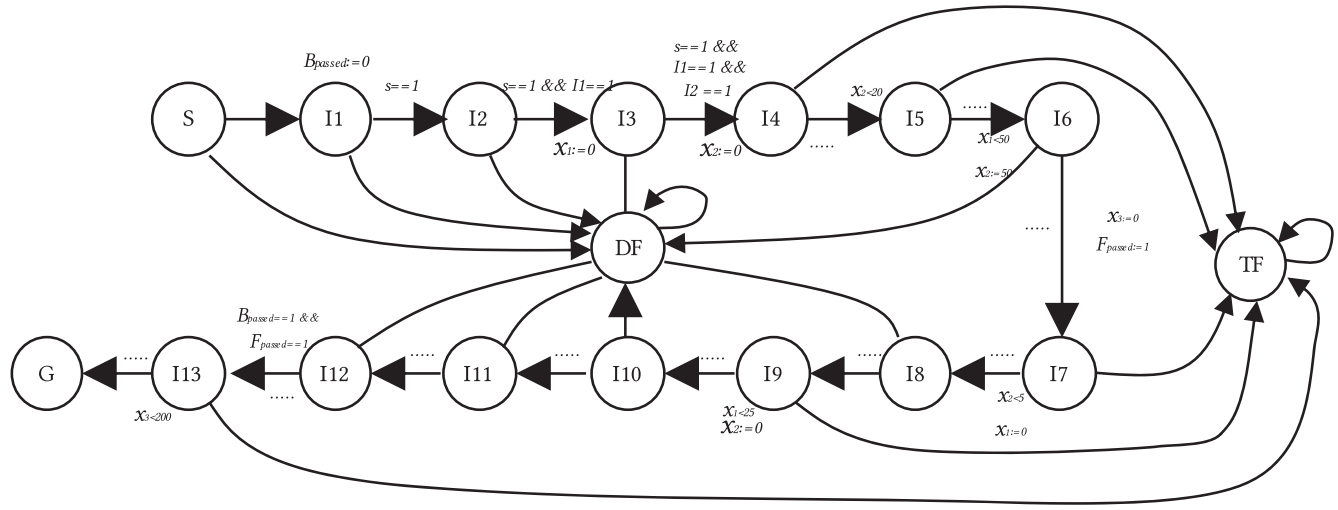

Fig. 16. UPPAAL Timed automata model for digital camera subsystem.

service routine after asserting the non-maskable interrupt. In contrast, the software-based SRM requires several hundred thousand cycles for detection. Additionally, as the target system does not allow ISRs to be interrupted, to generate the violation alert a software-based interrupt is asserted, which requires several hundred thousand cycles to start. In this example, the total detection latency for the SRM is $1,8327,90$ cycles, which is about $2 \mathrm{~ms}$.

\subsection{Comparison with Timed Automata Models}

The TA model is a well-known formal requirement and has been widely used in runtime verification methods. TA models can be expressed as states and transitions, and all state representations of the system are possible. Figure 16 shows an example TA model for a digital camera subsystem. To define dependency information in a multithreaded system, all dependency information must exist in the TA model. As a result, additional start and end states and failure states are required in the TA model, which involves more complicated conditional statements and thus more complicated TA models. In contrast, the RMG maintains the dependency of the operations using a more compact model representation, which is not possible in a TA model.

To compare the complexity of the RMG and TA models, we compare the number of vertices and edges within their respective graph models for the image capture and storage subsystem within a digital camera shown in Figure 2. For the TA model, we compare to a UPPALL timed-automata 
model automatically created from the UML sequence diagram (Firley et al. 1999). The resulting timed-automata model requires 31\% more vertices and 69\% more edges than the RMG to express the same set of timing and dependency requirements.

Finally, to compare the hardware requirements needed to verify a TA model, we developed programmable hardware to verify timed-automata models. The programmable hardware can be configured by software, which allows system designers to configure software requirements without re-implementing hardware. One of the challenges faced in developing the programmable TA model verifier is preserving variables for state transitions. For a system with $N$ states, the number of transitions and transition for each state can be as large as $N$. However, to keep hardware requirements low, we implemented a hardware prototype of the TA verifier that limits the number of transitions per state to three. Even with this limitation, the TA verifier requires significantly more area than the NIRM hardware. To support the digital camera subsystem, the TA verifier supports 17 states, requiring 2845 LUTs and 2501 FFs. For the same system, the NIRM hardware supports 11 events, requiring 1180 LUTs and $1685 \mathrm{FFs}$. Thus, the TA verifier required $1.8 \times$ more area to support the same system.

\section{CONCLUSIONS AND FUTURE WORK}

We presented a non-intrusive requirements monitoring methodology for automatically constructing a requirements model from UML sequence diagrams and verifying system correctness at runtime using non-intrusive on-chip in-situ hardware. The NIRM approach can achieve a $100 \%$ detection rate when monitoring only half of the full RMG. This proof-of-concept implementation demonstrates the feasibility of the proposed approach. Two case study system prototypes, namely an autonomous vehicle and smart connected pacemaker, demonstrate the efficiency of the NIRM approach for runtime verification in safety-critical and life-critical systems. We additionally presented several RMG optimization algorithms, demonstrating that multiphase iterative optimization algorithm provides an excellent tradeoff of $925 \times$ speedup with a reduction in detection rate of less than $2.3 \%$. Future work includes automated tools for extracting and optimizing the RMG considering energy constraints and developing efficient hardware capable of supporting hierarchical RMG to support larger scale requirements. Future work includes integration of hardware monitoring and verification with the presented software monitor and verification approach. These ongoing efforts include developing methods to automatically extract hardware verification requirements from state-based hardware models and integration of the resulting HRMGs for hardware and software components to create an integrated system-level HRMG. Future work also includes developing tools for optimizing path coverage of an RMG subject to energy constraints for the runtime verification and modeling of data-dependent timing and sequence requirements.

\section{REFERENCES}

Altera Verification Tool. 2006. SignalTap II Embedded Logic Analyzer. Retrieved from http://www.altera.com/products/ software/products/quartus2/verification/signaltap2/sig-index.html.

Rajeev Alur. 1999. Timed automata. In Computer Aided Verification. Springer, Berlin, 8-22. DOI: https://doi.org/10.1016/j. entcs.2008.12.102

Rico Backasch, Christian Hochberger, Alexander Weiss, Martin Leucker, and Richard Lasslop. 2013. Runtime verification for multicore soc with high-quality trace data. ACM Trans. Des. Autom. Electron. Syst. 18, 2 (March 2013), 1-26. DOI : https:// doi.org/10.1145/2442087.2442089

Tiller Beauchamp and David Weston. 2008. Dtrace: The Reverse Engineer's Unexpected Swiss Army Knife. Retrieved from http://www.poppopret.org/DTrace-Beauchamp-Weston.pdf.

Borzoo Bonakdarpour, Samaneh Navabpour, and Sebastian Fischmeister. 2011. Sampling-based runtime verification. In International Symposium on Formal Methods. Springer, 88-102. 
Matthias Brill, Werner Damm, Jochen Klose, Bernd Westphal, and Hartmut Wittke. 2004. Live sequence charts. In Springer, Berlin, 374-399. DOI : https://doi.org/10.1007/978-3-540-27863-4_21

Powell Douglass Bruce. 1999. Real-Time UML: Developing Efficient Objects for Embedded Systems (2nd. ed.). Addison-Wesley.

Ming Chai and Bernd-Holger Schlingloff. 2014. Monitoring systems with extended live sequence charts. In Proceedings of the International Conference on Runtime Verification. Springer, 48-63.

Alexandre David. 2003. Hierarchical Modeling and Analysis of Timed Systems. Ph.D. Dissertation. Uppsala University.

N. Delgado, A. Q. Gates, and S. Roach. 2004. A taxonomy and catalog of runtime software-fault monitoring tools. IEEE Trans. Softw. Eng. 30, 12 (Dec. 2004), 859-872. DOI : https://doi.org/10.1109/TSE.2004.91

Bruce Powel Douglass. 2014. Real-time UML Workshop for Embedded Systems. Newnes.

Thomas Firley, Michaela Huhn, Karsten Diethers, Thomas Gehrke, and Ursula Goltz. 1999. Timed sequence diagrams and tool-based analysis-a case study. In Proceedings of the International Conference on the Unified Modeling Language. Springer, 645-660.

Richard Fryer. 2005. FPGA based CPU instrumentation for hard real-time embedded system testing. ACM SIGBED Rev. 2 , 2 (2005), 39-42.

Sébastien Gérard and Bran Selic. 2008. The UML-MARTE Standardized profile. IFAC Proc. 41, 2 (2008), 6909-6913.

Brent Hailpern and Padmanabhan Santhanam. 2002. Software debugging, testing, and verification. IBM Syst. F. 41, 1 (2002), 4-12.

Richard Hofmann, Rainer Klar, Bernd Mohr, Andreas Quick, and Markus Siegle. 1994. Distributed performance monitoring: methods, tools, and applications. IEEE Trans. Parallel Distrib. Syst. 5, 6 (1994), 585-598.

Mike Jones. 1997. What really happened on mars rover pathfinder. Risks Dig. 19, 49 (1997), 1-2.

Aaron Kane, Omar Chowdhury, Anupam Datta, and Philip Koopman. 2015. A case study on runtime monitoring of an autonomous research vehicle (ARV) system. In Proceedings of the International Conference on Runtime Verification. Springer, 102-117.

Jong Chul Lee and Roman Lysecky. 2015. System-level observation framework for non-intrusive runtime monitoring of embedded systems. ACM Trans. Des. Autom. Electron. Syst. 20, 3 (Jun. 2015), 1-27. DOI : https://doi.org/10.1145/2717310

Nancy G. Leveson and Clark S. Turner. 1993. An investigation of the Therac-25 accidents. Computer 26, 7 (1993), $18-41$.

Hong Lu. 2007. The design and implementation of P2V, an architecture for zero-overhead online verification of software programs. Microsoft Research.

Shan Lu, Joseph Tucek, Feng Qin, and Yuanyuan Zhou. 2007. AVIO: Detecting atomicity violations via access-interleaving invariants. IEEE Micro 27, 1 (2007), 26-35. DOI : https://doi.org/10.1109/MM.2007.5

Kerryn Macaulay. 1992. ATSB preliminary factual report, in-flight upset, Qantas Airbus A330, $154 \mathrm{Km}$ West of Learmonth, WA, 7 October 2008. Australian Transport Safety Bureau Media Release, November 14 2008. (1992).

R. Mijat. 2010. Better trace for better software: Introducing the new arm coresight system trace macrocell and trace memory controller. ARM, White Paper (2010).

Niall Murphy and Michael Barr. 2001. Watchdog timers. Embed. Syst. Program. 14, 11 (2001), 79-80.

Ajay Nair and Roman Lysecky. 2008. Non-intrusive dynamic application profiler for detailed loop execution characterization. In Proceedings of the 2008 International Conference on Compilers, Architectures and Synthesis for Embedded Systems. ACM, 23-30.

Ajay Nair, Karthik Shankar, and Roman Lysecky. 2011. Efficient hardware-based nonintrusive dynamic application profiling. ACM Trans. Embed. Comput. Syst. 10, 3 (2011), 32.

Ahmed Nassar, Fadi J. Kurdahi, and Wael Elsharkasy. 2015. NUVA: Architectural support for runtime verification of parametric specifications over multicores. In Proceedings of the 2015 International Conference on Compilers, Architecture, and Synthesis for Embedded Systems (CASES'15), 137-146. DOI : https://doi.org/10.1109/CASES.2015.7324554

Samaneh Navabpour, Borzoo Bonakdarpour, and Sebastian Fischmeister. 2015. Time-triggered runtime verification of component-based multi-core systems. In Proceedings of the International Conference on Runtime Verification. Springer, 153-168.

Lee Pike, Sebastian Niller, and Nis Wegmann. 2011. Runtime verification for ultra-critical systems. In Proceedings of the International Conference on Runtime Verification. Springer, 310-324.

Amir Pnueli. 1977. The temporal logic of programs. In Proceedings of the 18th Annual Symposium on Foundations of Computer Science (SFCS'77). IEEE, 46-57. DOI : https://doi.org/10.1109/SFCS.1977.32

Thomas Reinbacher, Matthias Függer, and Jörg Brauer. 2012. Real-time runtime verification on chip. In Proceedings of the International Conference on Runtime Verification. Springer, 110-125.

James Rumbaugh, Ivar Jacobson, and Grady Booch. 2005. The Unified Modeling Language Reference Manual. AddisonWesley.

Mohammed El Shobaki. 2002. On-chip monitoring of single-and multiprocessor hardware real-time operating systems. In Proceedings of the 8th International Conference on Real-time Computing Systems and Applications (RTCSA'02).

Robert Skeel. 1992. Roundoff error and the patriot missile. SIAM News 25, 4 (1992), 11. 
Neal Stollon. 2011. On-Chip Instrumentation. Springer, Boston, MA.

Michael Whalen, Darren Cofer, Steven Miller, Bruce H. Krogh, and Walter Storm. 2007. Integration of formal analysis into a model-based software development process. In Formal Methods for Industrial Critical Systems. Springer, Berlin, 68-84. DOI : https://doi.org/10.1007/978-3-540-79707-4_7

Xilinx Verification Tool. 2006. ChipScope Pro. Retrieved from http://www.xilinx.com/ise/optional_prod/cspro.htm.

Pin Zhou, Radu Teodorescu, and Yuanyuan Zhou. 2007. HARD: Hardware-assisted lockset-based race detection. In Proceedings of the International Symposium on High-Performance Computing Architecture. 121-132. DOI : https://doi.org/10. 1109/HPCA.2007.346191

Received October 2017; revised February 2018; accepted May 2017 